\title{
LAPLACIAN GROWTH, SANDPILES, AND SCALING LIMITS
}

\author{
LIONEL LEVINE AND YUVAL PERES
}

\begin{abstract}
Laplacian growth is the study of interfaces that move in proportion to harmonic measure. Physically, it arises in fluid flow and electrical problems involving a moving boundary. We survey progress over the last decade on discrete models of (internal) Laplacian growth, including the abelian sandpile, internal DLA, rotor aggregation, and the scaling limits of these models on the lattice $\epsilon \mathbb{Z}^{d}$ as the mesh size $\epsilon$ goes to zero. These models provide a window into the tools of discrete potential theory, including harmonic functions, martingales, obstacle problems, quadrature domains, Green functions, smoothing. We also present one new result: rotor aggregation in $\mathbb{Z}^{d}$ has $O(\log r)$ fluctuations around a Euclidean ball, improving a previous power-law bound. We highlight several open questions, including whether these fluctuations are $O(1)$.
\end{abstract}

\section{The ABELIAN SANDPILE MODEL}

Start with $n$ particles at the origin in the square grid $\mathbb{Z}^{2}$, and let them spread out according to the following rule: whenever any site in $\mathbb{Z}^{2}$ has four or more particles, it gives one particle to each of its four nearest neighbors (north, east, south and west). The final configuration of particles does not depend on the order in which these moves are performed (which explains the term "abelian"; see Lemma 1.1 below).

This model was invented in 1987 by the physicists Bak, Tang and Wiesenfeld [7. While defined by a simple local rule, it produces self-similar global patterns that call for an explanation. Dhar [16] extended the model to any base graph and discovered the abelian property. The abelian sandpile was independently discovered by combinatorialists [10, who called it chip-firing. Indeed, in the last two decades the subject has been enriched by an exhilarating interaction of numerous areas of mathematics, including statistical physics, combinatorics, free boundary partial differential equations, probability, potential theory, number theory, and group theory. More on this below. There are also connections to algebraic geometry [8, 47, 56, commutative algebra [49,50, and computational complexity [6, 12, 52, For software for experimenting with sandpiles, see [57].

Let $G=(V, E)$ be a locally finite connected graph. A sandpile on $G$ is a function $s: V \rightarrow \mathbb{Z}$. We think of a positive value $s(x)>0$ as a number of sand grains (or particles) at vertex $x$, and a negative value as a hole that can be filled by particles. Vertex $x$ is unstable if $s(x) \geq \operatorname{deg}(x)$, the number of edges incident to

Received by the editors September 23, 2016.

2010 Mathematics Subject Classification. 31C20, 35R35, 60G50, 60K35, 82C24.

Key words and phrases. Abelian sandpile, chip-firing, discrete Laplacian, divisible sandpile, Eulerian walkers, internal diffusion limited aggregation, looping constant, obstacle problem, rotorrouter model, scaling limit, unicycle, Tutte slope.

The first author was supported by NSF grant DMS-1455272 and a Sloan Fellowship. 
$x$. Toppling $x$ is the operation of sending $\operatorname{deg}(x)$ particles away from $x$, one along each incident edge. We say that a sequence of vertices $\mathbf{x}=\left(x_{1}, \ldots, x_{m}\right)$ is legal for $s$ if $s_{i}\left(x_{i}\right) \geq \operatorname{deg}\left(x_{i}\right)$ for all $i=1, \ldots, m$, where $s_{i}$ is the sandpile obtained from $s$ by toppling $x_{1}, \ldots, x_{i-1}$; we say that $\mathbf{x}$ is stabilizing for $s$ if $s_{m} \leq \operatorname{deg}-1$. (All inequalities between functions are pointwise.)

Lemma 1.1. Let $s: V \rightarrow \mathbb{Z}$ be a sandpile, and suppose there exists a sequence $\mathbf{y}=\left(y_{1}, \ldots, y_{n}\right)$ that is stabilizing for $s$.

(i) Any legal sequence $\mathbf{x}=\left(x_{1}, \ldots, x_{m}\right)$ for $s$ is a permutation of a subsequence of $\mathbf{y}$.

(ii) There exists a legal stabilizing sequence for $s$.

(iii) Any two legal stabilizing sequences for s are permutations of each other.

Proof. Since $\mathbf{x}$ is legal for $s$, we have $s\left(x_{1}\right) \geq \operatorname{deg}\left(x_{1}\right)$. Since $\mathbf{y}$ is stabilizing for $s$, it follows that $y_{i}=x_{1}$ for some $i$. Toppling $x_{1}$ yields a new sandpile $s^{\prime}$. Removing $x_{1}$ from $\mathbf{x}$ and $y_{i}$ from $\mathbf{y}$ yields shorter legal and stabilizing sequences for $s^{\prime}$, so (i) follows by induction.

Let $\mathbf{x}$ be a legal sequence of maximal length, which is finite by (i). Such $\mathbf{x}$ must be stabilizing, which proves (ii).

Statement (iii) is immediate from (i).

We say that $s$ stabilizes if there is a sequence that is stabilizing for $s$. If $s$ stabilizes, we define its odometer as the function on vertices

$$
u(x)=\text { number of occurrences of } x \text { in any legal stabilizing sequence for } s .
$$

The stabilization $\hat{s}$ of $s$ is the result of toppling a legal stabilizing sequence for $s$. The odometer determines the stabilization, since

$$
\hat{s}=s+\Delta u
$$

where $\Delta$ is the graph Laplacian

$$
\Delta u(x)=\sum_{y \sim x}(u(y)-u(x)) .
$$

Here the sum is over vertices $y$ that are neighbors of $x$.

By Lemma 1.1(iii), both the odometer $u$ and the stabilization $\hat{s}$ depend only on $s$, and not on the choice of legal stabilizing sequence, which is one reason the model is called abelian (another is the role played by an abelian group; see Section 7).

What does a very large sandpile look like? The similarity of the two sandpiles in Figure 1 suggests that some kind of limit exists as we take the number of particles $n \rightarrow \infty$ while "zooming out" so that each square of the grid has area $1 / n$. The first step toward making this rigorous is to reformulate Lemma 1.1 in terms of the Laplacian as follows.

Least Action Principle. If there exists $w: V \rightarrow \mathbb{N}$ such that

$$
s+\Delta w \leq \operatorname{deg}-1,
$$

then $s$ stabilizes, and $w \geq u$ where $u$ is the odometer of $s$. Thus,

$$
u(x)=\inf \{w(x) \mid w: V \rightarrow \mathbb{N} \text { satisfies (3) }\} .
$$

Proof. If such $w$ exists, then any sequence $\mathbf{y}$ such that $w(x)=\#\left\{i: \mathbf{y}_{i}=x\right\}$ for all $x$ is stabilizing for $s$. The odometer is defined as $u(x)=\#\left\{i: \mathbf{x}_{i}=x\right\}$ for a 

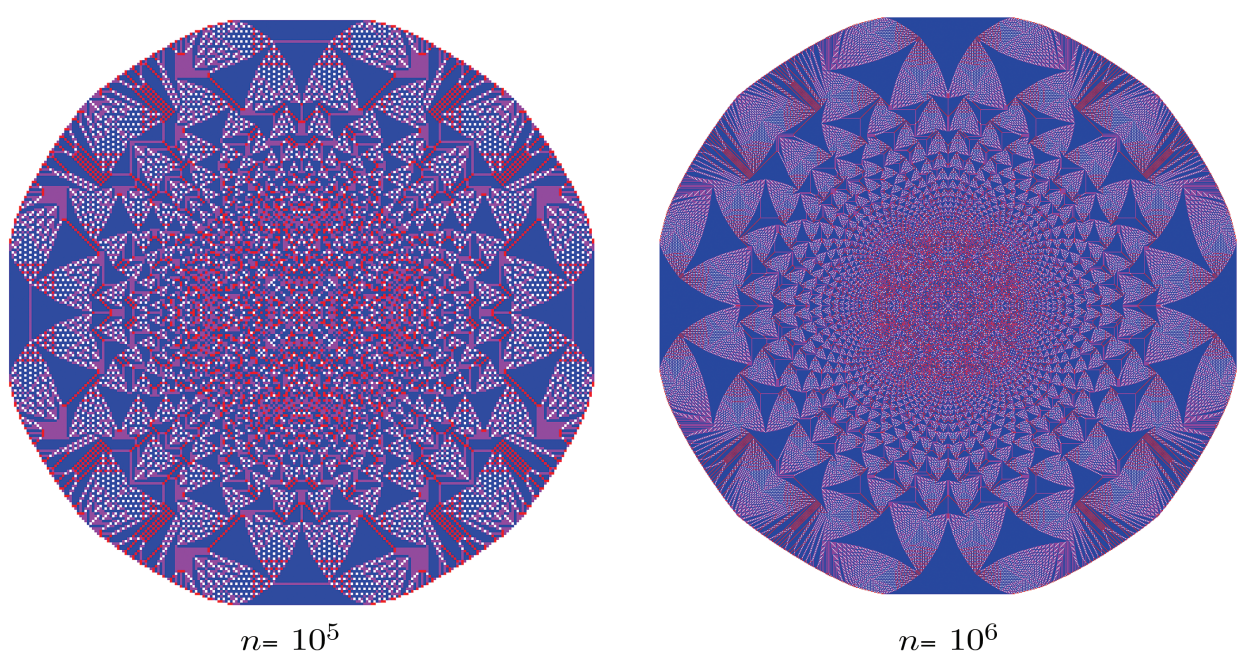

Figure 1 . Sandpiles in $\mathbb{Z}^{2}$ formed by stabilizing $10^{5}$ and $10^{6}$ particles at the origin. Each pixel is colored according to the number of sand grains that stabilize there (white 0 , red 1 , purple 2 , blue 3 ). The two images have been scaled to have the same diameter.

legal stabilizing sequence $\mathbf{x}$, so $w \geq u$ by part (i) of Lemma 1.1. The last line now follows from (11).

The Least Action Principle expresses the odometer as the solution to a variational problem (44). In the next section we will see that the same problem, without the integrality constraint on $w$, arises from a variant of the sandpile which will be easier to analyze.

\section{RELAXING INTEGRALity: THE DIVISIBLE SANDPILE}

Let $\mathbb{Z}^{d}$ be the set of points with integer coordinates in $d$-dimensional Euclidean space $\mathbb{R}^{d}$, and let $\mathbf{e}_{1}, \ldots, \mathbf{e}_{d}$ be its standard basis vectors. We view $\mathbb{Z}^{d}$ as a graph in which points $x$ and $y$ are adjacent if and only if $x-y= \pm \mathbf{e}_{i}$ for some $i$. For example, when $d=1$ this graph is an infinite path, and when $d=2$ it is an infinite square grid.

In the divisible sandpile model, each point $x \in \mathbb{Z}^{d}$ has a continuous amount of mass $\sigma(x) \in \mathbb{R}_{\geq 0}$ instead of a discrete number of particles. Start with mass $m$ at the origin and zero elsewhere. At each time step, choose a site $x \in \mathbb{Z}^{d}$ with mass $\sigma(x)>1$, where $\sigma$ is the current configuration, and distribute the excess mass $\sigma(x)-1$ equally among the $2 d$ neighbors of $x$. We call this a toppling. Suppose that these choices are sufficiently thorough in the sense that whenever a site attains mass $>1$, it is eventually chosen for toppling at some later time. Then we have the following version of the abelian property.

Lemma 2.1. For any initial $\sigma_{0}: \mathbb{Z}^{d} \rightarrow \mathbb{R}$ with finite total mass, and any thorough sequence of topplings, the mass function converges pointwise to a function $\sigma_{\infty}$ : $\mathbb{Z}^{d} \rightarrow \mathbb{R}$ satisfying $0 \leq \sigma_{\infty} \leq 1$. Any site $z$ satisfying $\sigma_{0}(z)<\sigma_{\infty}(z)<1$ has a neighboring site $y$ satisfying $\sigma_{\infty}(y)=1$. 
Proof. Let $u_{k}(x)$ be the total amount of mass emitted from $x$ during the first $k$ topplings, and let $\sigma_{k}=\sigma_{0}+\Delta u_{k}$ be the resulting mass configuration. Since $u_{k}$ is increasing in $k$, we have $u_{k} \uparrow u_{\infty}$ for some $u_{\infty}: V \rightarrow[0, \infty]$. To rule out the value $\infty$, consider the quadratic weight

$$
Q\left(\sigma_{k}\right):=\sum_{x \in \mathbb{Z}^{d}}\left(\sigma_{k}(x)-\sigma_{0}(x)\right)|x|^{2}=\sum_{x \in \mathbb{Z}^{d}} u_{k}(x) .
$$

To see the second equality, note that $Q$ increases by $h$ every time we topple mass $h$. The set $\left\{\sigma_{k} \geq 1\right\}$ is connected and contains $\mathbf{0}$, and has cardinality bounded by the total mass of $\sigma_{0}$, so it is bounded. Moreover, every site $z$ with $\sigma_{k}(z)>\sigma_{0}(z)$ has a neighbor $y$ with $\sigma_{k}(y) \geq 1$. Hence $\sup _{k} Q\left(\sigma_{k}\right)<\infty$, which shows that $u_{\infty}$ is bounded.

Finally, $\sigma_{\infty}:=\lim \sigma_{k}=\lim \left(\sigma_{0}+\Delta u_{k}\right)=\sigma_{0}+\Delta u_{\infty}$. By thoroughness, for each $x \in \mathbb{Z}^{d}$ we have $\sigma_{k}(x) \leq 1$ for infinitely many $k$, so $\sigma_{\infty} \leq 1$.

The picture is thus of a set of "filled" sites $\left(\sigma_{\infty}(z)=1\right)$ bordered by a strip of partially filled sites $\left(\sigma_{0}(z)<\sigma_{\infty}(z)<1\right)$. Every partially filled site has a filled neighbor, so the thickness of this border strip is only one lattice spacing. Think of pouring maple syrup over a waffle - most squares receiving syrup fill up completely and then begin spilling over into neighboring squares. On the boundary of the region of filled squares is a strip of squares that fill up only partially (Figure 3).

The limit $u_{\infty}$ is called the odometer of $\sigma_{0}$. The preceding proof did not show that $u_{\infty}$ and $\sigma_{\infty}$ are independent of the thorough toppling sequence. This is a consequence of the next result.

Least Action Principle for the divisible sandpile. For any $\sigma_{0}: \mathbb{Z}^{2} \rightarrow[0, \infty)$ with finite total mass and any $w: V \rightarrow[0, \infty)$ such that

$$
\sigma+\frac{1}{2 d} \Delta w \leq 1
$$

we have $w \geq u_{\infty}$ for any thorough toppling sequence. Thus,

$$
u_{\infty}(x)=\inf \{w(x): w: V \rightarrow[0, \infty) \text { satisfies (5) }\} .
$$

Proof. With the notation of the preceding proof, suppose for a contradiction that $u_{k} \not \leq w$ for some $k$. For the minimal such $k$, the functions $u_{k}$ and $u_{k-1}$ agree except at $x_{k}$, hence

$$
1=\left(\sigma+\frac{1}{2 d} \Delta u_{k}\right)\left(x_{k}\right)<\left(\sigma+\frac{1}{2 d} \Delta w\right)\left(x_{k}\right) \leq 1,
$$

which yields the required contradiction.

2.1. The superharmonic tablecloth. The variational problem (6) has an equivalent formulation:

Lemma 2.2. Let $\gamma: \mathbb{Z}^{d} \rightarrow \mathbb{R}$ satisfy $\frac{1}{2 d} \Delta \gamma=\sigma_{0}-1$. Then the odometer $u$ of (6) is given by

$$
u=s-\gamma,
$$

where

$$
s(x)=\inf \{f(x) \mid f \geq \gamma \text { and } \Delta f \leq 0\} .
$$

Proof. $f$ is in the set on the right side of (7) if and only if $w:=f-\gamma$ is in the set on the right side of ([6). 


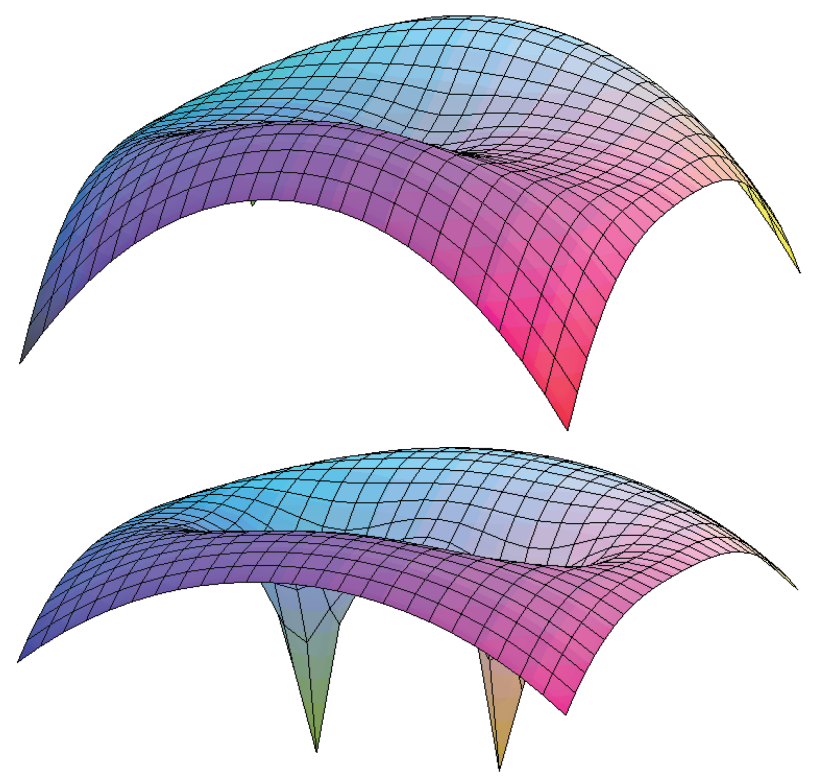

Figure 2. The obstacles $\gamma$ corresponding to starting mass 1 on each of two overlapping disks (top) and mass 100 on each of two nonoverlapping disks.

The function $\gamma$ is sometimes called the obstacle, and the minimizing function $s$ in (17) called the solution to the obstacle problem. To explain this terminology, we refer to Figure 2, which shows the graph of $\gamma$ for two different choices of initial mass configuration $\sigma_{0}$ on $\mathbb{Z}^{2}$. Imagine a graph of $\gamma$ as a fixed surface (for instance, the top of a table) and the graph of $f$ as a surface that can vary (a tablecloth). The tablecloth is constrained to stay above the table $(f \geq \gamma)$ and is further constrained to be superharmonic $(\Delta f \leq 0)$, which in particular implies that $f$ has no local minima. Depending on the shape of the table $\gamma$, these constraints may force the tablecloth to lie strictly above the table in some places.

The solution $s$ is the lowest possible position of the tablecloth. The set where strict inequality holds

$$
D:=\left\{x \in \mathbb{Z}^{d}: s(x)>\gamma(x)\right\}
$$

is called the noncoincidence set. In terms of the divisible sandpile, the odometer function $u$ is the gap $s-\gamma$ between tablecloth and table, and the set $\{u>0\}$ of sites that topple is the noncoincidence set.

2.2. Building the obstacle. The reader ought now to be wondering, given a configuration $\sigma_{0}: \mathbb{Z}^{d} \rightarrow[0, \infty)$ of finite total mass, what the corresponding obstacle $\gamma: \mathbb{Z}^{d} \rightarrow \mathbb{R}$ looks like. The only requirement on $\gamma$ is that it has a specified discrete Laplacian, namely

Does such $\gamma$ always exist?

$$
\frac{1}{2 d} \Delta \gamma=\sigma_{0}-1
$$

Given a function $f: \mathbb{Z}^{d} \rightarrow \mathbb{R}$, we would like to construct a function $F$ such that $\Delta F=f$. The most straightforward method is to assign arbitrary values for $F$ on a 
pair of parallel hyperplanes, from which the relation $\Delta F=f$ determines the other values of $F$ uniquely.

This method suffers from the drawback that the growth rate of $F$ is hard to control. A better method uses what is called the Green function or fundamental solution for the discrete Laplacian $\Delta$. This is a certain function $g: \mathbb{Z}^{d} \rightarrow \mathbb{R}$ whose discrete Laplacian is zero except at the origin:

$$
\frac{1}{2 d} \Delta g(x)=-\delta_{\mathbf{0}}(x)= \begin{cases}-1 & x=\mathbf{0}, \\ 0 & x \neq \mathbf{0} .\end{cases}
$$

If $f$ has finite support, then we can construct $F$ as a convolution

$$
F(x)=-f * g:=-\sum_{y \in \mathbb{Z}^{d}} f(y) g(x-y)
$$

in which only finitely many terms are nonzero. (The condition that $f$ has finite support can be relaxed to fast decay of $f(x)$ as $|x| \rightarrow \infty$, but we will not pursue this.) Then for all $x \in \mathbb{Z}^{d}$, we have

$$
\Delta F(x)=\sum_{y \in \mathbb{Z}^{d}} f(y) \delta_{\mathbf{0}}(x-y)=f(x)
$$

as desired. By controlling the growth rate of the Green function $g$, we can control the growth rate of $F$. The minus sign in equation (8) is a convention: as we will now see, with this sign convention $g$ has a natural definition in terms of random walk.

Let $\xi_{1}, \xi_{2}, \ldots$ be a sequence of independent random variables each with the uniform distribution on the set $\mathcal{E}=\left\{ \pm \mathbf{e}_{1}, \ldots, \pm \mathbf{e}_{d}\right\}$. For $x \in \mathbb{Z}^{d}$, the sequence

$$
X_{n}=\xi_{1}+\cdots+\xi_{n}, \quad n \geq 0,
$$

is called a simple random walk started from the origin in $\mathbb{Z}^{d}$ : it is the location of a walker who has wandered from $\mathbf{0}$ by taking $n$ independent random steps, choosing each of the $2 d$ coordinate directions $\pm \mathbf{e}_{i}$ with equal probability $1 / 2 d$ at each step.

In dimensions $d \geq 3$ the simple random walk is transient: its expected number of returns to the origin is finite. In these dimensions we define

$$
g(x):=\sum_{n \geq 0} \mathbb{P}\left(X_{n}=x\right),
$$

a function known as the Green function of $\mathbb{Z}^{d}$. It is the expected number of visits to $x$ by a simple random walk started at the origin in $\mathbb{Z}^{d}$. The identity

$$
-\frac{1}{2 d} \Delta g=\delta_{0}
$$

is proved by conditioning on the first step $X_{1}$ of the walk

$$
\begin{aligned}
g(x) & =P\left(X_{0}=x\right)+\sum_{n \geq 1} \sum_{e \in \mathcal{E}} P\left(X_{n}=x \mid X_{1}=e\right) P\left(X_{1}=e\right) \\
& =\delta_{\mathbf{0}}(x)+\sum_{n \geq 1} \sum_{e \in \mathcal{E}} P\left(X_{n-1}=x-e\right) \frac{1}{2 d} .
\end{aligned}
$$

Interchanging the order of summation, the second term on the right equals $\frac{1}{2 d} \sum_{y \sim x} g(y)$, and (9) now follows by the definition of the Laplacian $\Delta$. 
The case $d=2$ is more delicate because the simple random walk is recurrent: with probability 1 it visits $x$ infinitely often, so the sum defining $g(x)$ diverges. In this case, $g$ is defined instead as

$$
g(x)=\sum_{n \geq 0}\left(\mathbb{P}\left(X_{n}=x\right)-\mathbb{P}\left(X_{n}=\mathbf{0}\right)\right) .
$$

One can show that this sum converges and that the resulting function $g: \mathbb{Z}^{2} \rightarrow \mathbb{R}$ satisfies (9); see 66]. The function $-g$ is called the recurrent potential kernel of $\mathbb{Z}^{2}$.

Convolving with the Green function enables us to construct functions on $\mathbb{Z}^{d}$, whose discrete Laplacian is any given function with finite support. But we want more: In Lemma 2.2 we seek a function $\gamma$ satisfying $\Delta \gamma=\sigma-1$, where $\sigma$ has finite support. Fortunately, there is a very nice function whose discrete Laplacian is a constant function, namely the squared Euclidean norm

$$
q(x)=|x|^{2}:=\sum_{i=1}^{d} x_{i}^{2} .
$$

(In fact, we implicitly used the identity $\frac{1}{2 d} \Delta q \equiv 1$ in the quadratic weight argument for Lemma 2.1) We can therefore take as our obstacle the function

$$
\gamma=-q-(g * \sigma) .
$$

In order to determine what happens when we drape a superharmonic tablecloth over this particular table $\gamma$, we should figure out what $\gamma$ looks like! In particular, we would like to know the asymptotic order of the Green function $g(x)$ when $x$ is far from the origin. It turns out [22, 36, 67, that

$$
g(x)=G(x)+O\left(|x|^{-d}\right),
$$

where $G$ is the spherically symmetric function

$$
G(x):= \begin{cases}-\frac{2}{\pi} \log |x|-a_{2}, & d=2 ; \\ a_{d}|x|^{2-d}, & d \geq 3 .\end{cases}
$$

(For $d \geq 3$ the constant $a_{d}=\frac{2}{(d-2) \omega_{d}}$, where $\omega_{d}$ is the volume of the unit ball in $\mathbb{R}^{d}$. The constant $a_{2}=\frac{2 \gamma+\log 8}{\pi}$, where $\gamma$ is Euler's constant.) As we will now see, this estimate in combination with $-\frac{1}{2 d} \Delta g=\delta_{\mathbf{0}}$ is a powerful package. We start by analyzing the initial condition $\sigma=m \delta_{\mathbf{0}}$ for large $m$.

2.3. Point sources. Pour $m$ grams of maple syrup into the center square of a very large waffle. Each square can hold just 1 gram of syrup before it overflows, distributing the excess equally among the four neighboring squares. What is the shape of the resulting set of squares that fill up with syrup?

Figure 3 suggests the answer is very close to a disk. Being mathematicians, we wish to quantify "very close", and why stop at two-dimensional waffles? Let $B(\mathbf{0}, r)$ be the Euclidean ball of radius $r$ centered at the origin in $\mathbb{R}^{d}$.

Theorem 2.3 (44]). Let $D_{m}=\left\{\sigma_{\infty}=1\right\}$ be the set of fully occupied sites for the divisible sandpile started from mass $m$ at the origin in $\mathbb{Z}^{d}$. There is a constant $c=c(d)$, such that

$$
B(\mathbf{0}, r-c) \cap \mathbb{Z}^{d} \subset D_{m} \subset B(\mathbf{0}, r+c),
$$




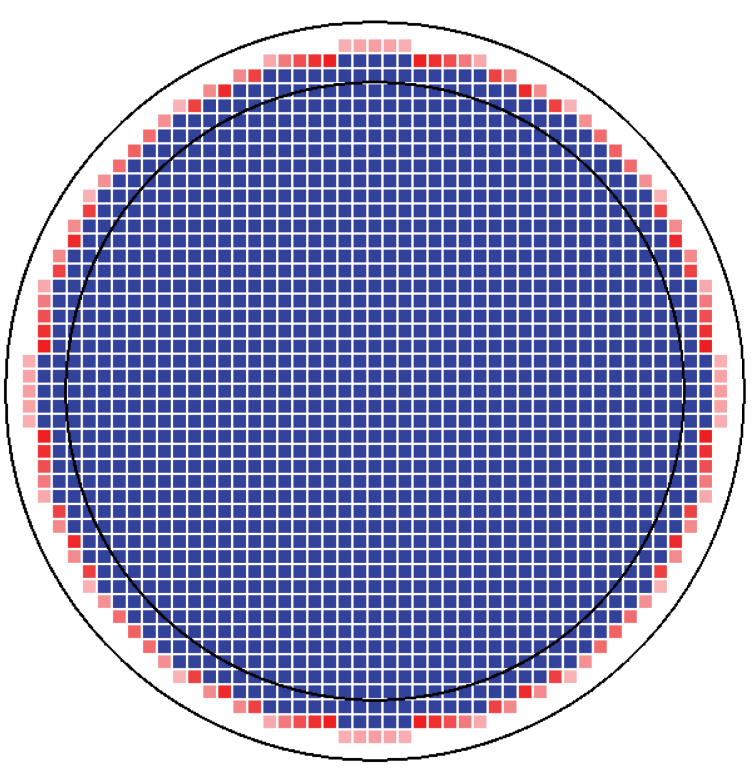

Figure 3. Divisible sandpile in $\mathbb{Z}^{2}$ started from mass $m=1600$ at the origin. Each square is colored blue if it fills completely, red if it fills only partially. All red squares are contained in a thin annulus centered at the origin of radii $r \pm c$ where $\pi r^{2}=m$. This is illustrated above with $c=2$.

where $r$ is such that $B(\mathbf{0}, r)$ has volume $m$. Moreover, the odometer $u_{\infty}$ satisfies

$$
u_{\infty}(x)=m g(x)+|x|^{2}-m g\left(r \mathbf{e}_{1}\right)-r^{2}+O(1)
$$

for all $x \in B(\mathbf{0}, r+c) \cap \mathbb{Z}^{d}$, where the constant in the $O$ depends only on $d$.

The idea of the proof is to use Lemma 2.2 to write the odometer function as

$$
u_{\infty}=s-\gamma
$$

for an obstacle $\gamma$ with discrete Laplacian $\frac{1}{2 d} \Delta \gamma=m \delta_{\mathbf{0}}-1$. What does such an obstacle look like?

Recalling that the Euclidean norm $|x|^{2}$ and the discrete Green function $g$ have discrete Laplacians 1 and $-\delta_{\mathbf{0}}$, respectively, a natural choice of obstacle is

$$
\gamma(x)=-|x|^{2}-m g(x) .
$$

The claim of (13) is that $u(x)$ is within an additive constant of $\gamma\left(r \mathbf{e}_{1}\right)-\gamma(x)$. To prove this, one uses two properties of $\gamma$ : it is nearly spherically symmetric (because $g$ is!) and it is maximized near $|x|=r$. From these properties one deduces that $s$ is nearly a constant function, and that $\{s>\gamma\}$ is nearly the ball $B(\mathbf{0}, r) \cap \mathbb{Z}^{d}$.

The Euclidean ball as a limit shape is an example of universality: Although our topplings took place on the cubic lattice $\mathbb{Z}^{d}$, if we take the total mass $m \rightarrow \infty$ while zooming out so that the cubes of the lattice become infinitely small, the divisible sandpile assumes a perfectly spherical limit shape. Figure 1 strongly suggests that the abelian sandpile, with its indivisible grains of sand, does not enjoy such universality. However, discrete particles are not incompatible with universality, as the next two examples show. 


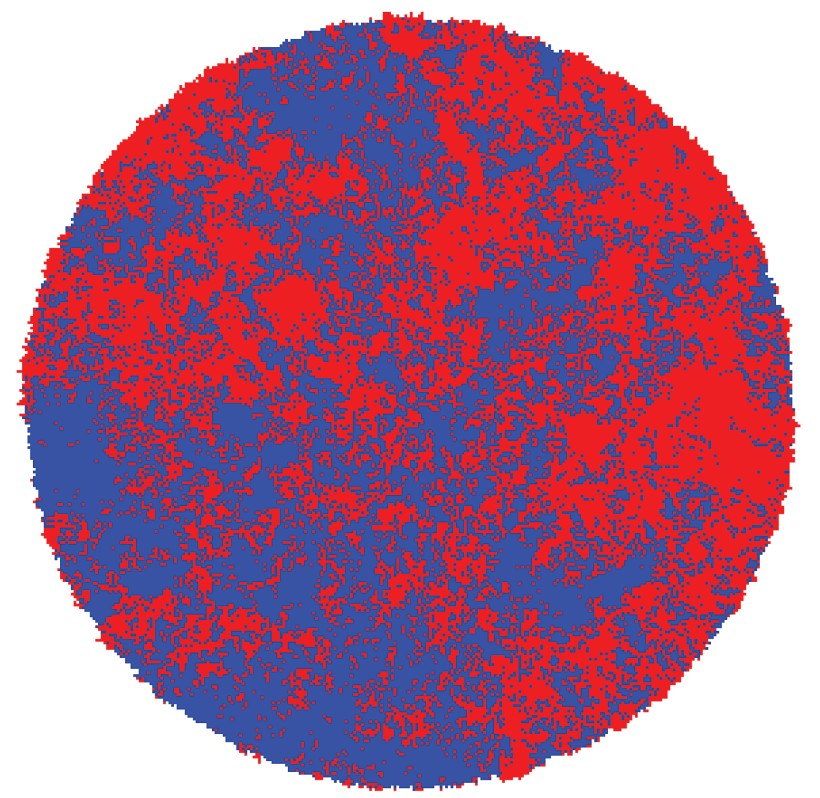

Figure 4. An internal DLA cluster in $\mathbb{Z}^{2}$. The colors indicate whether a point was added to the cluster earlier or later than expected: the random site $x(j)$ where the $j$ th particle stops is colored red if $\pi|x(j)|^{2}>j$, blue otherwise.

\section{INTERNAL DLA}

Let $m \geq 1$ be an integer. Starting with $m$ particles at the origin in the $d$ dimensional integer lattice $\mathbb{Z}^{d}$, let each particle in turn perform a simple random walk until reaching an unoccupied site; that is, the particle repeatedly jumps to a nearest neighbor chosen independently and uniformly at random, until it lands on a site containing no other particles.

This procedure, known as internal DLA, was proposed by Meakin and Deutch [51] and independently by Diaconis and Fulton [19]. It produces a random set $I_{m}$ of $m$ occupied sites in $\mathbb{Z}^{d}$ (Figure 4). This random set is close to a ball, in the following sense. Let $r$ be such that the Euclidean ball $B(\mathbf{0}, r)$ of radius $r$ has volume $m$. Lawler, Bramson, and Griffeath [40] proved that for any $\epsilon>0$, with probability 1 it holds that

$$
B(\mathbf{0},(1-\epsilon) r) \cap \mathbb{Z}^{d} \subset I_{m} \subset B(\mathbf{0},(1+\epsilon) r) \quad \text { for all sufficiently large } m \text {. }
$$

A sequence of improvements followed, showing that in dimensions $d \geq 2$ the fluctuations of $I_{m}$ around $B(\mathbf{0}, r)$ are logarithmic in $r[2,4,29,31,38$.

\section{Rotor-Routing: DERANDOMizED RANDOM WALK}

In a rotor walk on a graph, each vertex $v$ serves its neighbors in a prescribed periodic sequence. This periodic sequence is called the rotor mechanism at $v$. We say that the rotor mechanism at $v$ is simple if each neighbor of $v$ occurs exactly once per period. To visualize a rotor walk, label each vertex by an arrow (rotor) pointing toward one of its neighbors. At each time step, the walker first advances the rotor 


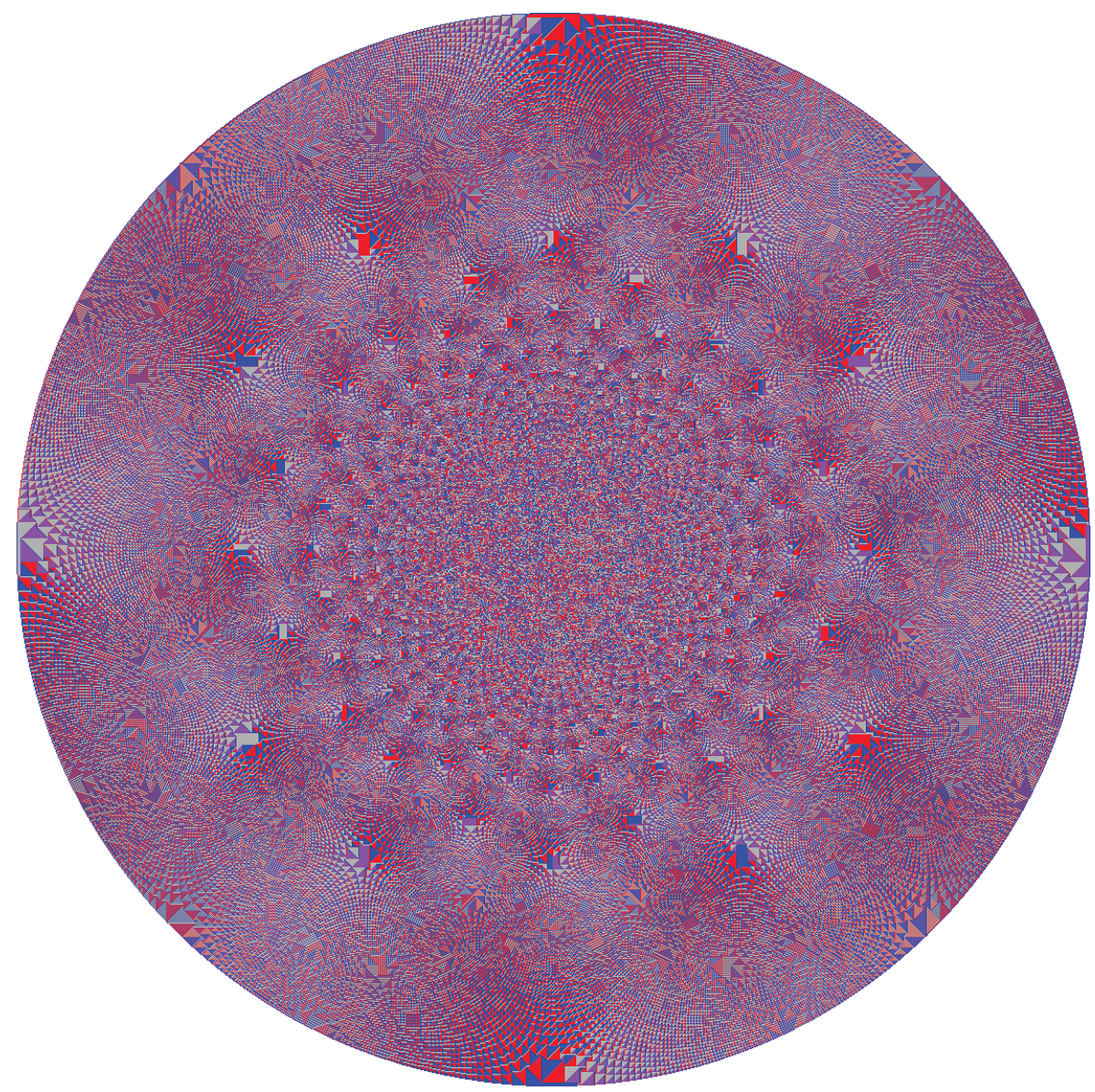

FiguRE 5. Rotor aggregate of one million particles started at the origin in $\mathbb{Z}^{2}$. All rotors initially pointed north and followed the clockwise mechanism: each rotor repeatedly cycles through the directions north, east, south, west. Each pixel represents a site of $\mathbb{Z}^{2}$, and its color indicates the final direction of its rotor.

at its current location to point to the next neighbor in the periodic sequence, and then the walker moves to that neighbor.

Rotor walk has been studied in 68 as a model of mobile agents exploring a territory, and in 61] as a model of self-organized criticality. Propp 63. proposed rotor walk as a derandomization of random walk, a perspective explored in [15,26].

In rotor aggregation, we start with $n$ walkers at the origin; each in turn performs rotor-router walk until it reaches a site not occupied by any other walkers. Importantly, we do not reset the rotors between walks! Let $R_{n}$ denote the resulting region of $n$ occupied sites. For example, in $\mathbb{Z}^{2}$ with the clockwise rotor mechanism whose fundamental period is north, east, south, west, the sequence will begin $R_{1}=\{\mathbf{0}\}$, $R_{2}=\left\{\mathbf{0}, \mathbf{e}_{1}\right\}, R_{3}=\left\{\mathbf{0}, \mathbf{e}_{1},-\mathbf{e}_{2}\right\}$. The region $R_{10^{6}}$ is pictured in Figure 5 ,

Theorem 4.1. There is a constant $C$ depending only on the dimension $d$, such that for any initial rotor configuration and any simple rotor mechanism on $\mathbb{Z}^{d}$, the 
rotor aggregate $R_{n}$ formed from $n=\left\lfloor\omega_{d} r^{d}\right\rfloor$ particles started at $\mathbf{0}$ satisfies

$$
B(\mathbf{0}, r-C \log r) \cap \mathbb{Z}^{d} \subset R_{n} \subset B(\mathbf{0}, r+C \log r) .
$$

The inner bound was proved in [4], which also included a weaker outer bound $R_{\omega_{d} r^{d}} \subset B\left(\mathbf{0}, r+C r^{(d-1) / d} \log r\right)$. The rest of this section is devoted to the proof of the stronger outer bound (15), which builds on ideas of Holroyd and Propp [26] and Jerison, Levine, and Sheffield [29,30].

4.1. No thin tentacles. The first step in the proof of Theorem 4.1 is to rule out "thin tentacles" on the boundary of the rotor aggregate $R_{n}$. Namely, according to the following proposition, for any point $z_{0} \in R_{n}$ at least a constant fraction of the lattice sites in a ball around $z_{0}$ must also belong to $R_{n}$. An analogous result for internal DLA appears in [29, Lemma A].

Proposition 4.2 (No thin tentacles). There is a positive constant c depending only on the dimension $d$, such that for any initial rotor configuration and any simple rotor mechanism in $\mathbb{Z}^{d}$, and for any $z_{0} \in R_{n}$ and $\rho<\left|z_{0}\right|$, we have

$$
\#\left(B\left(z_{0}, \rho\right) \cap R_{n}\right) \geq c \rho^{d} .
$$

The proof of Proposition 4.2 uses the odometer function $u=u_{n}: \mathbb{Z}^{d} \rightarrow \mathbb{N}$ defined by

$u(x):=$ total number of exits from $x$ by all $n$ particles during rotor aggregation.

If the same particle exits $x$ several times, then we include all of its exits in the count.

We first compare the gradient of $u$ with the net number of crossings of an edge. For each directed edge $(x, y)$ of $\mathbb{Z}^{d}$, let

$$
\theta(x, y):=N(x, y)-N(y, x)
$$

where $N(x, y)$ is the total number of exits from $x$ to $y$ by all $n$ particles in rotorrouter aggregation.

Lemma 4.3. For all directed edges $(x, y)$ of $\mathbb{Z}^{d}$,

$$
u(x)-u(y)=2 d \theta(x, y)+\beta(x, y)
$$

for a function $\beta$ on directed edges of $\mathbb{Z}^{d}$ which satisfies

$$
|\beta(x, y)| \leq 4 d-2 \text {. }
$$

Proof. Note that $u(x)=\sum_{y \sim x} N(x, y)$. There are $2 d$ terms in this sum, and the definition of a simple rotor mechanism implies that any two of them differ by at most 1 , so for $x \sim y$,

$$
|u(x)-2 d N(x, y)| \leq 2 d-1 .
$$

By the triangle inequality,

$$
\begin{aligned}
|u(x)-u(y)-2 d \theta(x, y)| & \leq|u(x)-2 d N(x, y)|+|u(y)-2 d N(y, x)| \\
& \leq 4 d-2 .
\end{aligned}
$$

Next consider the discrete Laplacian of the odometer function $u$. Since the net effect of all rotor moves is to transport $n$ particles at the origin to one particle at each site of $R_{n}$, we would like to say that " $\frac{1}{2 d} \Delta u=1_{R_{n}}-n \delta_{\mathbf{0}}$ on average". (This equality holds exactly in a special case: namely, if every rotor makes an integer number of full turns, so that $N(x, y)$ depends only on $x$, then $\frac{1}{2 d} \Delta u(x)$ equals the 
total number of entries to $x$ minus the total number of exits from $x$.) One way to make a precise statement of this form is to smooth $u$ by averaging its values over a small ball

$$
\mathbb{B}(x, k):=\mathrm{B}(x, k) \cap \mathbb{Z}^{d} .
$$

The proof of the next lemma is a discrete version of $\Delta=\operatorname{div} \nabla$.

Lemma 4.4. Fix an integer $k>1$. For $f: \mathbb{Z}^{d} \rightarrow \mathbb{R}$, write

$$
S_{k} f(x):=\frac{1}{\# \mathbb{B}(x, k)} \sum_{y \in \mathbb{B}(x, k)} f(y) .
$$

For all $x$ such that $\mathbb{B}(x, k) \subset R_{n}-\{\mathbf{0}\}$, the odometer $u=u_{n}$ satisfies

$$
\frac{1}{2 d} \Delta S_{k} u(x)=1+O\left(\frac{1}{k}\right)
$$

where the implied constant depends only on the dimension $d$.

Proof. Note that $\Delta S_{k}=S_{k} \Delta$. Hence

$$
\frac{1}{2 d} \Delta S_{k} u(x)=\frac{1}{\# \mathbb{B}(x, k)} \sum_{y \in \mathbb{B}(x, k)} \frac{1}{2 d} \Delta u(y) .
$$

Writing the Laplacian $\Delta u(y)$ as $\sum_{z \sim y}(u(z)-u(y))$, we see that the interior terms with $y, z \in Q$ cancel, leaving (by Lemma 4.3)

$$
\frac{1}{\# \mathbb{B}(x, k)} \sum_{y \in \mathbb{B}(x, k), z \notin \mathbb{B}(x, k), z \sim y}\left(\theta(z, y)+\frac{1}{2 d} \beta(z, y)\right) .
$$

The sum of the $\theta$ terms is the net number of particles entering $\mathbb{B}(x, k)$, which is exactly $\# \mathbb{B}(x, k)$ : the ball starts empty and ends with exactly one particle per site, since $\mathbb{B}(x, k) \subset R_{n}-\{\mathbf{0}\}$. Each $\beta$ term is $O(1)$ by Lemma 4.3 , and there are $O\left(k^{d-1}\right)$ terms in all (one for each boundary edge of $\left.\mathbb{B}(x, k)\right)$. Dividing by $\# \mathbb{B}(x, k)$ leaves $1+O(1 / k)$.

The above proof without any smoothing gives something much cruder. Namely, if $x \in \mathbb{Z}^{d}-\{\mathbf{0}\}$, then the net number of particles entering $x$ is $\sum_{y} \theta(y, x)=1_{x \in R_{n}}$, so

$$
\left|\frac{1}{2 d} \Delta u(x)-1_{x \in R_{n}}\right|=\left|\frac{1}{2 d} \sum_{y \sim x}(u(y)-u(x)-2 d \theta(y, x))\right| \leq 4 d-2
$$

by Lemma 4.3. In particular,

$$
|\Delta u| \leq 8 d^{2} \quad \text { on } \mathbb{Z}^{d}-\{\mathbf{0}\} .
$$

We will use Lemma 4.4 for a fixed $k=k(d)$, chosen large enough so that

$$
\left|\frac{1}{2 d} \Delta S_{k} u(x)-1\right|<\frac{1}{4}
$$

for all $x$ such that $\mathbb{B}(x, k) \subset R_{n}-\{\mathbf{0}\}$.

Next we record the following lemma, which is proved by applying the Harnack inequality to the harmonic extensions of the functions $f(x) \pm|x|^{2}$ in $\mathbb{B}(\mathbf{0}, r)$; see the proof of Lemma 2.17 in section 7 of [45]. 
Lemma 4.5. Fix $r>1$, and let $f$ be a nonnegative function on $\mathbb{Z}^{d}$ satisfying $f(\mathbf{0})=0$ and $|\Delta f| \leq 1$ on $\mathbb{B}(\mathbf{0}, 2 r)$. There is a constant $A_{0}$ depending only on $d$, such that

$$
f(x) \leq A_{0}|x|^{2}
$$

for all $x \in \mathbb{B}(\mathbf{0}, r) \cap \mathbb{Z}^{d}$.

The next lemma shows that if the odometer is large at $x$, then the cluster $R_{n}$ contains a ball centered at $x$.

Lemma 4.6. Let $A=8 d^{2} A_{0}$. If $u(x)>A k^{2}$ and $|x|>3 k$, then $\mathbb{B}(x, k) \subset R_{n}$.

Proof. We prove the contrapositive: If $\mathbb{B}(x, k) \not \subset R_{n}$, then there is a $y \in \mathbb{B}(x, k)$ such that $u(y)=0$. Since $u \geq 0$, and $\Delta u$ is bounded by $8 d^{2}$ on $\mathbb{B}(y, 2 k)$ (here we use that $\mathbf{0} \notin \mathbb{B}(x, 3 k))$, it follows from Lemma 4.5 that $u(x) \leq A k^{2}$.

Lemma 4.7. If $S_{k} u(x)>4 A k^{2}$ and $|x|>7 k$, then $\mathbb{B}(x, k) \subset R_{n}$.

Proof. Since $S_{k} u(x)$ is the average of $u$ over $\mathbb{B}(x, k)$, there exists $x^{\prime} \in \mathbb{B}(x, k)$ with $u\left(x^{\prime}\right) \geq S_{k} u(x)$. Now Lemma 4.6 implies that $\mathbb{B}(x, k) \subset \mathbb{B}\left(x^{\prime}, 3 k\right) \subset R_{n}$.

The next two lemmas follow [45. Lemmas 4.9 and 4.10]. For $x \in \mathbb{Z}^{d}$ and $\rho>0$, let

$$
N(x, \rho):=\#\left(\mathbb{B}(x, \rho) \cap R_{n}\right)
$$

be the number of occupied sites within distance $\rho$ of $x$.

Lemma 4.8. Fix $x \in R_{n}$ and $\rho<|x|$. Let $m:=\max _{\partial \mathbb{B}(x, \rho)}$ u. Then $N(x, \rho+1) \geq$ $\left(1+\frac{1}{m}\right) N(x, \rho)$.

Proof. Note that $m \geq 1$ since $x \in R_{n}$. Since $\rho<|x|$, no particles start in $\mathbb{B}(x, \rho)$. Each particle entering $\mathbb{B}(x, \rho)$ must pass through the external vertex boundary $\partial \mathbb{B}(x, \rho)$, so

$$
N(x, \rho) \leq \sum_{y \in \partial \mathbb{B}(x, \rho)} u(y) .
$$

The sum on the right has at most $N(x, \rho+1)-N(x, \rho)$ nonzero terms, since $\partial \mathbb{B}(x, \rho) \subset \mathbb{B}(x, \rho+1)-\mathbb{B}(x, \rho)$. Each term is at most $m$, so

$$
N(x, \rho) \leq m N(x, \rho+1)-m N(x, \rho) .
$$

Fix $k$ large enough that (18) holds, and let

$$
R_{n, k}=\left\{x \in \mathbb{Z}^{d}: \mathbb{B}(x, k) \subset R_{n}\right\} .
$$

Lemma 4.9. There are constants $n_{0}$ and $\rho_{0}$ depending only on $d$, such that for all $n>n_{0}$ the following holds. For each $z_{0} \in R_{n}$ there exists $z_{1} \in R_{n, k}$ with $\left|z_{0}-z_{1}\right|<\rho_{0}$ and $S_{k} u\left(z_{1}\right)>4 A k^{2}$. (Recall that $\left.u=u_{n}.\right)$

Proof. Since $n$ particles start at $\mathbf{0}$, we have $u(\mathbf{0}) \geq n-1$. Choose $n_{0}$ large enough that $S_{k} u(\mathbf{0})>4 A k^{2}$. Take $\rho_{0}:=D k^{d+3}$ where the constant $D$ is chosen below. If $\left|z_{0}\right| \leq \rho_{0}$ then take $z_{1}=\mathbf{0}$. Otherwise, setting $m:=\max _{\mathbb{B}\left(z_{0}, \rho_{0}\right)} u$, we iteratively apply Lemma 4.8 to obtain

$$
\left(1+\frac{1}{m}\right)^{\rho_{0}} \leq N\left(z_{0}, \rho_{0}\right)
$$


(here we have used that $z_{0} \in R_{n}$ so that $N\left(z_{0}, 0\right)=1$ and $m \geq 1$ ). By definition, the right side is at most $\# \mathbb{B}\left(z_{0}, \rho_{0}\right)$. Taking the logarithm of both sides yields

$$
\frac{\rho_{0}}{m} \leq C \log \rho_{0}
$$

for a constant $C$ depending only on $d$. Taking $D$ large enough in the choice of $\rho_{0}$ above, it follows that $m>4 A k^{2} \# \mathbb{B}\left(z_{0}, k\right)$, so there exists $z_{1} \in \mathbb{B}\left(z_{0}, \rho\right)$ with $S_{k} u\left(z_{1}\right)>4 A k^{2}$. By Lemma 4.6 it follows that $z_{1} \in R_{n, k}$.

Lemma 4.9 shows that near each point in $R_{n}$ is a point in $R_{n, k}$. Next we show that for each point in $R_{n, k}$ there is a nearby point with large odometer. The proof is by the maximum principle, using an idea of Caffarelli [11.

Lemma 4.10. If $S_{k} u\left(z_{1}\right)>4 A k^{2}$, then for every $\rho$ satisfying $k<\rho<\left|z_{1}\right|-4 k$, there exists $z_{2} \in \mathbb{B}\left(z_{1}, \rho\right)$ such that

$$
S_{k} u\left(z_{2}\right)>\frac{1}{2} \rho^{2} .
$$

Proof. Note that for every $x \in \mathcal{A}:=\mathbb{B}\left(z_{1}, \rho\right) \cap R_{n, k}$, we have $\mathbb{B}(x, k) \subset R_{n}-\{\mathbf{0}\}$, so by (18) the function

$$
f(x):=S_{k} u(x)-\frac{1}{2}\left|x-z_{1}\right|^{2}
$$

is subharmonic in $\mathcal{A}$. By the maximum principle,

$$
\max _{x \in \mathcal{A} \cup \partial \mathcal{A}} f(x)=\max _{x \in \partial \mathcal{A}} f(x),
$$

where $\partial \mathcal{A}:=\left\{x \in \mathcal{A}^{c}: x \sim y\right.$ for some $\left.y \in \mathcal{A}\right\}$. Note that $z_{1} \in \mathcal{A}$, and for all $x \in R_{n, k}^{c} \cap \partial \mathcal{A}$, Lemma 4.7 implies that

$$
f(x) \leq S_{k} u(x) \leq 4 A k^{2}<f\left(z_{1}\right),
$$

so the maximum (19) must be attained at some $x \in R_{n, k} \cap \partial \mathcal{A}$. It follows that $x \notin \mathbb{B}\left(z_{1}, \rho\right)$, so

$$
S_{k} u(x)>\frac{1}{2}\left|x-z_{1}\right|^{2}>\frac{1}{2} \rho^{2} .
$$

Proof of Proposition 4.2. Fix $k=k(d)$ so that (18) holds. By taking $c$ sufficiently small, we may assume that $\rho>\rho_{0}$, where $\rho_{0}$ was defined in Lemma 4.9. By that lemma, there is a point $z_{1} \in R_{n, k}$ with $\left|z_{1}-z_{0}\right|<\rho / 4$. Now by Lemma 4.10 there is a point $z_{2}$ with $S_{k} u\left(z_{2}\right)>\frac{1}{2} \rho^{2}$ and $\left|z_{2}-z_{1}\right|<\rho / 2$. It follows by Lemma 4.7 that $R_{n}$ contains a ball $\mathbb{B}\left(z_{2}, \rho / C\right)$. Since this ball has volume $c \rho^{d}$, and $\left|z_{2}-z_{0}\right|<3 \rho / 4$, the proof is complete.

4.2. The Holroyd-Propp bound; probe functions. If $h$ is a function on $\mathbb{Z}^{d}$ and $A \subset \mathbb{Z}^{d}$ is a finite set, write $h[A]:=\sum_{x \in A} h(x)$.

Lemma 4.11 (Holroyd-Propp bound, [26]). For any initial rotor configuration and any simple rotor mechanism on $\mathbb{Z}^{d}$, if $h: \mathbb{Z}^{d} \rightarrow \mathbb{R}$ is discrete harmonic on $R_{n}$, then

$$
\left|h\left[R_{n}\right]-n h(\mathbf{0})\right| \leq \sum_{x \in R_{n}} \sum_{y \sim x}|h(x)-h(y)| .
$$


Proof. For each vertex $v \in R_{n}$, let $v_{1}, \ldots, v_{2 d}$ be the neighbors of $v$ in $\mathbb{Z}^{d}$ in the order they appear in $v$ 's rotor mechanism. We assign a weight $w_{i}(v) \in \mathbb{R}$ to a rotor pointing from $v$ to $v_{i}$, so that $w_{1}(v)=0$ and

$$
w_{i}(v)-w_{i-1}(v)=h(v)-h\left(v_{i}\right)
$$

for each $i=1, \ldots, 2 d$ (taking indices modulo $2 d$ ). These assignments are consistent since $h$ is discrete harmonic: $\sum_{i}\left(h(v)-h\left(v_{i}\right)\right)=0$. We also assign weight $h(v)$ to a walker located at $v$. The sum of rotor and walker weights is unchanged by each step of the rotor walk. Initially, the sum of all walker weights is $n h(\mathbf{0})$. After all walkers have stopped, the sum of all walker weights is $h\left[R_{n}\right]$. Their difference is thus at most the change in rotor weights, which is bounded above by the right side of (20).

Next we describe our choice of discrete harmonic function $h$, which is a variant of those used in $[29,30$. The idea is that for each point $y$ slightly outside $\mathbb{B}(\mathbf{0}, r)$, we can build a discrete harmonic probe function $h=h_{y}$ whose sum $h\left[R_{n}\right]$ measures how close the cluster $R_{n}$ comes to $y$. A good choice of $h$ turns out to be a discrete derivative of Green's function in the radial direction: the essential properties of this $h$ are that it is nonnegative in a neighborhood of the ball $\mathbb{B}(\mathbf{0}, r)$ and it decays rapidly away from $y$.

In the next lemma, $J, K, L, M$ are constants depending only on the dimension $d$.

Lemma 4.12 (Probe function). Fix $r>\rho>J$. For each $y \in \mathbb{Z}^{d}$ with $r+2 \rho<$ $|y|<r+3 \rho$, there exists a function $h: \mathbb{Z}^{d} \rightarrow \mathbb{R}$ with the following properties.

(i) $h$ is discrete harmonic and nonnegative on $\mathbb{B}(\mathbf{0}, r+\rho)$.

(ii) $h(x)<K|x-y|^{1-d}$.

(iii) $h(x)>\frac{1}{4} \rho^{1-d}$ for all $x \in \mathbb{B}(\mathbf{0}, r+\rho) \cap \mathbb{B}(y, 2 \rho)$.

(iv) $\sum_{x \in \mathbb{B}(\mathbf{0}, r+\rho)} \sum_{z \sim x}|h(x)-h(z)|<L \log r$.

(v) $h[\mathbb{B}(\mathbf{0}, r)]>(\# \mathbb{B}(\mathbf{0}, r)) h(\mathbf{0})-M \log r$.

Proof. Let

$$
h(x):=b \sum_{i=1}^{d} \frac{y_{i}}{|y|}\left[g\left(x-y+\mathbf{e}_{i}\right)-g(x-y)\right],
$$

where $g$ is the Green function of $\mathbb{Z}^{d}$ defined in Section 2.2 and $b$ is a small constant we will choose later.

Since $g$ is discrete harmonic on $\mathbb{Z}^{d}-\{\mathbf{0}\}$, the function $h$ is discrete harmonic on $\mathbb{Z}^{d}-\mathbb{B}(y, 1)$. To show that $h$ is nonnegative and to prove (ii)-(v), we approximate $g$ by its continuum analogue $G$ of (11), which yields

$$
h(x)=b \sum_{i=1}^{d} \frac{y_{i}}{|y|}\left[G\left(x-y+\mathbf{e}_{i}\right)-G(x-y)\right]+O\left(|x-y|^{-d}\right) .
$$

Next observe that

$$
\frac{\partial G}{\partial x_{i}}(x)=-\frac{2}{\omega_{d}} \frac{x_{i}}{|x|^{d}}
$$

in all dimensions $d \geq 2$, and the second derivatives of $G$ are $O\left(|x|^{-d}\right)$. (We remark that $G$ also has a simple physical interpretation as the Newtonian potential of a 
mass at the origin in $\mathbb{R}^{d}$. The gradient of $G$ is the gravitational force exerted by a mass at $\mathbf{0}$ on a mass at $x$.) A first-order Taylor expansion of $G$ around $x-y$ gives

$$
h(x)=b \sum_{i=1}^{d} \frac{y_{i}}{|y|}\left[\frac{2}{\omega_{d}} \frac{y_{i}-x_{i}}{|y-x|^{d}}\right]+O\left(|y-x|^{-d}\right),
$$

where the implied constant in the error term depends only on $d$. Take $b=\omega_{d} / 2$ so that

$$
h(x)=\frac{\langle y, y-x\rangle}{|y||y-x|}|y-x|^{1-d}+O\left(|y-x|^{-d}\right) .
$$

(i) Setting $z=(r+\rho) \frac{y}{|y|}$, we have for all $x \in B(\mathbf{0}, r+\rho)$

$$
\langle y, y-x\rangle \geq\langle y, y-z\rangle>\rho|y|
$$

so that

$$
h(x)>(\rho-O(1))|y-x|^{-d}>0
$$

on $B(\mathbf{0}, r+\rho)$ (take $J$ large enough to beat the $O(1))$.

(ii) By Cauchy-Schwarz inequality, the prefactor in equation (22) is at most 1.

(iii) For $x \in \mathbb{B}(y, 2 \rho) \cap \mathbb{B}(\mathbf{0}, r+\rho)$, we have by (22) and (23)

$$
h(x)>\frac{\rho|y|}{2 \rho|y|} \rho^{1-d}-O\left(\rho^{-d}\right) .
$$

Take $J>4$ to ensure that (iii) holds.

(iv) This follows from (11).

(v) Writing $H(x)$ for the main term of (21), we have

$$
\int_{B(\mathbf{0}, r)} h(x) \mathrm{d} x=\int_{B(\mathbf{0}, r)} H(x) \mathrm{d} x+O(\log r)=\omega_{d} r^{d} H(\mathbf{0})+O(\log r) .
$$

Write $B^{\square}$ for the union of closed unit cubes centered at the points of $\mathbb{B}(\mathbf{0}, r)$. Then

$$
\sum_{x \in \mathbb{B}(\mathbf{0}, r)} h(s)=\int_{B^{\square}} H(x) \mathrm{d} x+O(\log r) .
$$

Moreover, since $h(x)<K|x-y|^{1-d}$, we have $\int_{B} \square-\int_{B(\mathbf{0}, r)} H=O(\log r)$.

Now we prove the main result of this section. As in 29, 30 the idea is to amplify the ability of $h\left[R_{n}\right]$ to detect fluctuations in $R_{n}$ : The absence of thin tentacles (Proposition 4.2) implies that if $R_{n}$ has a point within distance $\rho$ of $y$, then $R_{n}$ has a substantial number of points (at least $c \rho^{d}$ ) within distance $2 \rho$ of $y$. Each of these points contributes substantially to the sum $h\left[R_{n}\right]$, but if $\rho$ is on the order of $\log r$, then $c \rho^{d}$ is very small compared to $n$. To show that their contribution is not swamped by the rest of $R_{n}$, we use the inner bound of (15) and the discrete mean value property Lemma $4.12(\mathrm{v})$.

Proof of Theorem 4.1. The inner bound was proved in [44. To prove the outer bound, let

$$
\rho:=\max \left(J, \max _{x \in R_{n}}|x|-r\right) .
$$

By 44 we have $\rho<r$ (in fact $\rho<r^{(d-1) / d} \log r$ ).

We may assume that $\rho>J$. Fix $y \in \partial \mathbb{B}(\mathbf{0}, r+2 \rho)$ such that $\mathbb{B}(y, 3 \rho / 2)$ intersects $R_{n}$, and let $h$ be as in Lemma 4.12. By Proposition 4.2 $\#\left(R_{n} \cap B(y, 2 \rho)\right) \geq c \rho^{d}$. 
Since $R_{n} \subset B(\mathbf{0}, r+\rho)$ and $|y|>r+2 \rho$, it follows that $R_{n} \cap B(y, 2 \rho) \subset A:=$ $B(y, 2 \rho)-B(y, \rho)$. By Lemma 4.12(iii) we have

$$
h\left[R_{n} \cap A\right]>\left(\frac{1}{4} \rho^{1-d}\right)\left(c \rho^{d}\right)=\frac{c}{4} \rho .
$$

By the discrete mean value property, Lemma $4.12(\mathrm{v})$, writing $n_{1}=\# \mathbb{B}(\mathbf{0}, r-C \log r)$ we have

$$
h[\mathbb{B}(\mathbf{0}, r-C \log r)]>n_{1} h(\mathbf{0})-M \log r .
$$

By Lemma 4.12(i) we have $h \geq 0$ on $R_{n}$. Now we throw away the terms $h(x)$ for $x \notin A \cup B(\mathbf{0}, r-C \log r)$ and use the inner bound $\mathbb{B}(\mathbf{0}, r-C \log r) \subset R_{n}$, obtaining

$$
h\left[R_{n}\right]-n h(\mathbf{0})>h\left[R_{n} \cap A\right]-M \log r-\left(n-n_{1}\right) h(\mathbf{0}) .
$$

The left side is at most $L \log r$, by the Holroyd-Propp Lemma 4.11 and Lemma 4.12 (iv). Using $n_{1}>(r-2 C \log r)^{d}$, we get

$$
(L+M) \log r>\frac{c}{4} \rho-\left(2 C d r^{d-1} \log r\right) h(\mathbf{0}) .
$$

Finally, since $h(\mathbf{0})<K r^{1-d}$ by Lemma 4.12 (ii), we conclude that

$$
\rho<C^{\prime} \log r
$$

as desired, with $C^{\prime}=\frac{4}{c}(2 C K d+L+M)$.

\section{Multiple Sources; Quadrature domains}

The Euclidean ball as a limiting shape is not too hard to guess. But what if the particles start at two different points of $\mathbb{Z}^{d}$ ? For example, fix an integer $r \geq 1$ and a positive real number $a$, and start with $m=\left\lfloor\omega_{d}(a r)^{d}\right\rfloor$ particles at each of $r \mathbf{e}_{1}$ and $-r \mathbf{e}_{1}$. Alternately release a particle from $r \mathbf{e}_{1}$ and let it perform a simple random walk until it finds an unoccupied site, and then release a particle from $-r \mathbf{e}_{1}$ and

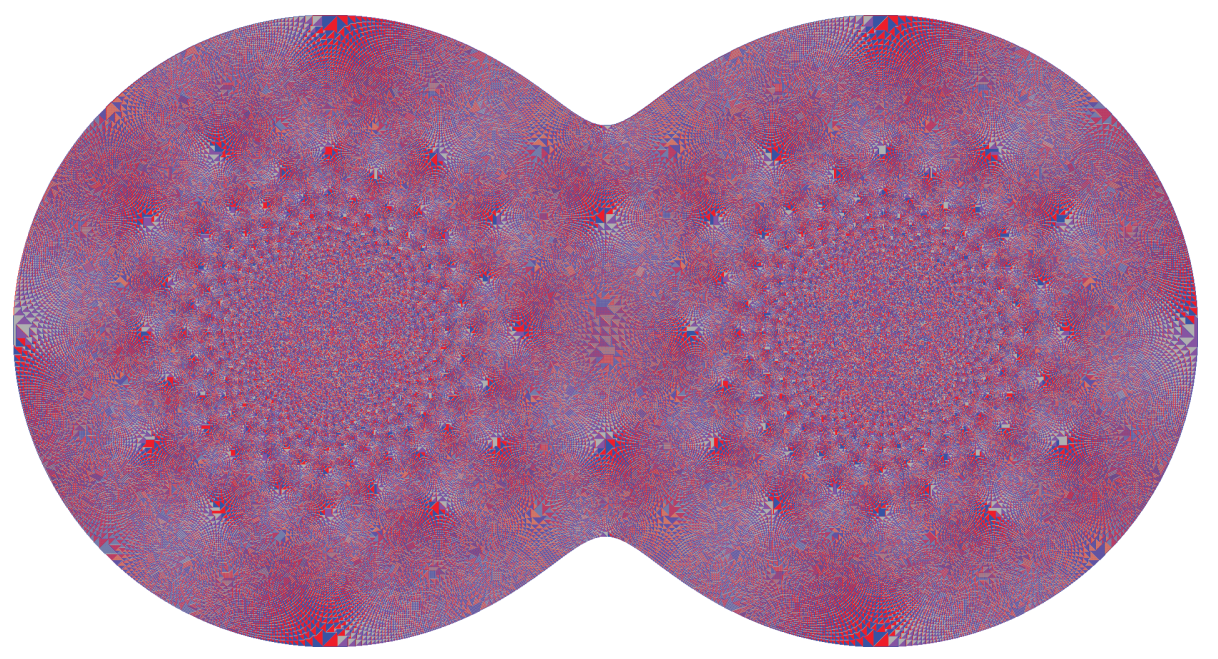

FIGURE 6. Rotor-router aggregation started from two-point sources in $\mathbb{Z}^{2}$. Its scaling limit is a two-point quadrature domain in $\mathbb{R}^{2}$, satisfying (26). 
let it perform a simple random walk until it finds an unoccupied site. The result is a random set $I_{m, m}$ consisting of $2 m$ occupied sites in $\mathbb{Z}^{d}$.

If $a<1$, then the distance between the source points $\pm r \mathbf{e}_{1}$ is so large compared to the number of particles that, with high probability, the particles starting at $r \mathbf{e}_{1}$ do not interact with those starting at $-r \mathbf{e}_{1}$. In this case $I_{m, m}$ is a disjoint union of two ball-shaped clusters each of size $m$. On the other hand, if $a \gg 1$, so that the two source points are very close together relative to the number of particles released, then the cluster $I_{m, m}$ will look like a single ball of size $2 m$. What happens in between these extreme cases?

Theorem 5.1 (45]). For each $a>0$ there exists a deterministic domain $D \subset \mathbb{R}^{d}$ such that with probability 1

$$
\frac{1}{r} I_{m, m} \rightarrow D
$$

as $r \rightarrow \infty$.

The precise meaning of the convergence of domains in (24) is the following. Given $D_{r} \subset \frac{1}{r} \mathbb{Z}^{d}$ and $\Omega \subset \mathbb{R}^{d}$, we write $D_{r} \rightarrow \Omega$ if for all $\epsilon>0$ we have

$$
\Omega_{\epsilon} \cap \frac{1}{r} \mathbb{Z}^{d} \subset D_{r} \subset \Omega^{\epsilon}
$$

for all sufficiently large $r$, where

$$
\Omega_{\epsilon}:=\{x \in \Omega \mid \overline{\mathrm{B}}(x, \epsilon) \subset \Omega\}
$$

and

$$
\Omega^{\epsilon}:=\left\{x \in \mathbb{R}^{d} \mid \mathrm{B}(x, \epsilon) \not \subset \Omega^{c}\right\}
$$

are the inner and outer $\epsilon$-neighborhoods of $D$.

The limiting domain $D$ is called a quadrature domain because it satisfies

$$
\frac{1}{\omega_{d} a^{d}} \int_{D} h \mathrm{~d} x=h\left(-\mathbf{e}_{1}\right)+h\left(\mathbf{e}_{1}\right)
$$

for all integrable harmonic functions $h$ on $D$, where $\mathrm{d} x$ is the Lebesgue measure on $\mathbb{R}^{d}$. This identity is analogous to the mean value property $\int_{B} h \mathrm{~d} x=h(\mathbf{0})$ for integrable harmonic functions on the ball $B$ of unit volume centered at the origin.

In dimension $d=2$, the domain $D$ has a more explicit description: For $a \geq 1$ its boundary in $\mathbb{R}^{2}$ is the quartic curve

$$
\left(x^{2}+y^{2}\right)^{2}-2 a^{2}\left(x^{2}+y^{2}\right)-2\left(x^{2}-y^{2}\right)=0 .
$$

When $a=1$ this factors as

$$
\left(x^{2}+y^{2}-2 x\right)\left(x^{2}+y^{2}+2 x\right)=0,
$$

which describes the union of two unit circles centered at $\pm \mathbf{e}_{1}$ and tangent at the origin. This case corresponds to two clusters that just barely interact, whose interaction is small enough that we do not see it in the limit. When $a \gg 1$, the term $2\left(x^{2}-y^{2}\right)$ is much smaller than the others, so the curve (27) is approximately the circle

$$
x^{2}+y^{2}-2 a^{2}=0 .
$$

This case corresponds to releasing so many particles that the effect of releasing them alternately at $\pm r e_{1}$ is nearly the same as releasing them all at the origin. An intermediate example, with $a$ slightly larger than 1, is shown in Figure 6.

Theorem 5.1 extends to the case of any $k$-point sources in $\mathbb{R}^{d}$ as follows. 
Theorem 5.2 ([45]). Fix $x_{1}, \ldots, x_{k} \in \mathbb{R}^{d}$ and $a_{1}, \ldots, a_{k}>0$. Let $x_{i}^{:}$be a closest site to $x_{i}$ in the lattice $\frac{1}{n} \mathbb{Z}^{d}$, and let

$$
\begin{aligned}
D_{n} & =\{\text { occupied sites for the divisible sandpile }\}, \\
R_{n} & =\{\text { occupied sites for rotor aggregation }\}, \\
I_{n} & =\{\text { occupied sites for internal } D L A\},
\end{aligned}
$$

started in each case from $\left\lfloor a_{i} n^{d}\right\rfloor$ particles at each site $x_{i}^{:}$in $\frac{1}{n} \mathbb{Z}^{d}$.

Then there is a deterministic set $D \subset \mathbb{R}^{d}$ such that

$$
D_{n}, R_{n}, I_{n} \rightarrow D,
$$

where the convergence is in the sense of (25), the convergence for $R_{n}$ holds for any initial setting of the rotors, and the convergence for $I_{n}$ is with probability 1 .

The limiting set $D$ is called a $k$-point quadrature domain. It is characterized up to measure zero by the inequalities

$$
\int_{D} h \mathrm{~d} x \leq \sum_{i=1}^{k} a_{i} h\left(x_{i}\right)
$$

for all integrable superharmonic functions $h$ on $D$, where $\mathrm{d} x$ is the Lebesgue measure on $\mathbb{R}^{d}$. The subject of quadrature domains in the plane begins with Aharonov and Shapiro [1] and was developed by Gustafsson [24, Sakai 64,65, and others. The boundary of a $k$-point quadrature domain in the plane lies on an algebraic curve of degree $2 k$. In dimensions $d \geq 3$, it is not known whether the boundary of $D$ is an algebraic surface!

\section{SCAling Limit of the ABELian SANDPile ON $\mathbb{Z}^{2}$}

Now that we have seen an example of a universal scaling limit, let us return to our very first example, the abelian sandpile with discrete particles.

Take as our underlying graph the square grid $\mathbb{Z}^{2}$, start with $n$ particles at the origin, and stabilize. The resulting configuration of sand appears to be noncircular (Figure 1) - so we do not expect the scaling limit to be universal like the one in Theorem 5.2. In a breakthrough work [54, Pegden and Smart proved existence of its scaling limit as $n \rightarrow \infty$. To state their result, let

$$
s_{n}=n \delta_{\mathbf{0}}+\Delta u_{n}
$$

be the sandpile formed from $n$ particles at the origin in $\mathbb{Z}^{d}$, and consider the rescaled sandpile

$$
\bar{s}_{n}(x)=s_{n}\left(n^{1 / d} x\right) .
$$

Theorem 6.1 ([54]). There is a function $s: \mathbb{R}^{d} \rightarrow \mathbb{R}$ such that $\bar{s}_{n} \rightarrow s$ is weakly-* in $L^{\infty}\left(\mathbb{R}^{d}\right)$.

The weak-* convergence of $\bar{s}_{n}$ in $L^{\infty}$ means that for every ball $B(x, r)$, the average of $s_{n}$ over $\mathbb{Z}^{d} \cap n^{1 / d} B(x, r)$ tends as $n \rightarrow \infty$ to the average of $s$ over $B(x, r)$.

The limiting sandpile $s$ is lattice dependent. Examining the proof in 54 reveals that the lattice dependence enters in the following way. Each real symmetric $d \times d$ matrix $A$ defines a quadratic function $q_{A}(\mathbf{x})=\frac{1}{2} \mathbf{x}^{T} A \mathbf{x}$ and an associated sandpile $s_{A}: \mathbb{Z}^{d} \rightarrow \mathbb{Z}$

$$
s_{A}=\Delta\left\lceil q_{A}\right\rceil .
$$




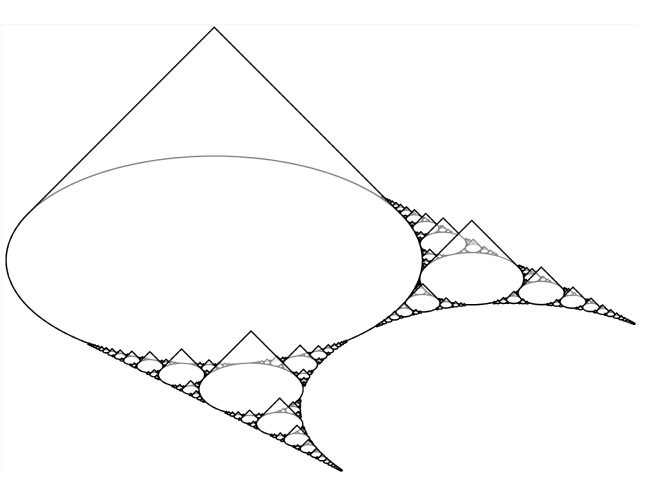

(i)

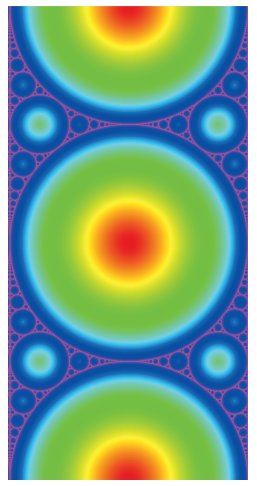

(ii)

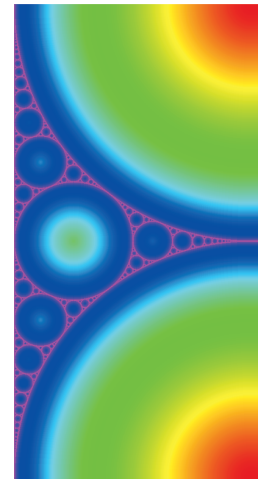

(iii)

Figure 7. (i) According to the main theorem of [43, the set of allowed Hessians $\Gamma\left(\mathbb{Z}^{2}\right)$ is the union of slope 1 cones based at the circles of an Apollonian circle packing in the plane of $2 \times 2$ real symmetric matrices of trace 2 . (ii) The same set viewed from above: Color of point $(a, b)$ indicates the largest $c$ such that $\left(\begin{array}{cc}c-a & b \\ b & c+a\end{array}\right) \in \Gamma\left(\mathbb{Z}^{2}\right)$. The rectangle shown, $(a, b) \in[0,2] \times[0,4]$, extends periodically to the entire plane. (iii) Close-up of the lower left corner $(a, b) \in[0,1] \times[0,2]$.

For each matrix $A$, the sandpile $s_{A}$ either stabilizes locally (that is, every site of $\mathbb{Z}^{d}$ topples finitely often), or it fails to stabilize (in which case every site topples infinitely often). The set of allowed Hessians $\Gamma\left(\mathbb{Z}^{d}\right)$ is defined as the closure (with respect to the Euclidean norm $\left.\|A\|_{2}^{2}=\operatorname{Tr}\left(A^{T} A\right)\right)$ of the set of matrices $A$ such that $s_{A}$ stabilizes locally.

One can convert the Least Action Principle into an obstacle problem analogous to Lemma 2.2 with an additional integrality constraint. The limit of these discrete obstacle problems on $\frac{1}{n} \mathbb{Z}^{d}$ as $n \rightarrow \infty$ is the following variational problem on $\mathbb{R}^{d}$.

\section{Limit of the Least Action Principle.}

$$
u=\inf \left\{w \in C\left(\mathbb{R}^{d}\right) \mid w \geq-G \text { and } D^{2}(w+G) \in \Gamma\left(\mathbb{Z}^{d}\right)\right\} .
$$

Here $G$ is the fundamental solution of the Laplacian in $\mathbb{R}^{d}$. The infimum is pointwise, and the minimizer $u$ is related to the the sandpile odometers $u_{n}$ by

$$
\lim _{n \rightarrow \infty} \frac{1}{n} u_{n}\left(n^{1 / 2} x\right)=u(x)+G(x) .
$$

The Hessian constraint in (28) is interpreted in the sense of viscosity:

$$
D^{2} \varphi(x) \in \Gamma\left(\mathbb{Z}^{d}\right)
$$

whenever $\varphi$ is a $C^{\infty}$ function touching $w+G$ from below at $x$ (that is, $\varphi(x)=$ $w(x)+G(x)$ and $\varphi-(w+G)$ has a local maximum at $x)$.

The obstacle $G$ in (28) is a spherically symmetric function on $\mathbb{R}^{d}$, so the lattice dependence arises solely from $\Gamma\left(\mathbb{Z}^{d}\right)$. Put another way, the set $\Gamma\left(\mathbb{Z}^{d}\right)$ is a way of quantifying which features of the lattice $\mathbb{Z}^{d}$ are still detectable in the limit of sandpiles as the lattice spacing shrinks to zero. 
An explicit description of $\Gamma\left(\mathbb{Z}^{2}\right)$ appears in [4] (see Figure 7), and explicit fractal solutions of the sandpile PDE

$$
D^{2} u \in \partial \Gamma\left(\mathbb{Z}^{2}\right)
$$

are constructed in [42. See [55] for images of $\Gamma(L)$ for some other two-dimensional lattices $L$.

\section{THE SANDPILE GROUP OF A FINITE GRAPH}

Let $G=(V, E)$ be a finite connected graph, and fix a sink vertex $z \in V$. A stable sandpile is now a map $s: V \backslash\{z\} \rightarrow \mathbb{N}$ satisfying $s(x)<\operatorname{deg}(x)$ for all $x \in V \backslash\{z\}$. As before, sites $x$ with $s(x) \geq \operatorname{deg}(x)$ topple by sending one particle along each edge incident to $x$, but now particles falling into the sink disappear.

Define a Markov chain on the set of stable sandpiles as follows. At each time step, add one sand grain at a vertex of $V \backslash\{z\}$ selected uniformly at random, and then perform all possible topplings until the sandpile is stable. Recall that a state $s$ in a finite Markov chain is called recurrent if whenever $s^{\prime}$ is reachable from $s$, then $s$ is also reachable from $s^{\prime}$. Dhar [16] observed that the operation $a_{x}$ of adding one particle at vertex $x$ and then stabilizing is a permutation of the $\operatorname{set} \operatorname{Rec}(G, z)$ of recurrent sandpiles. These permutations obey the relations

$$
a_{x} a_{y}=a_{y} a_{x} \quad \text { and } \quad a_{x}^{\operatorname{deg}(x)}=\prod_{u \sim x} a_{u}
$$

for all $x, y \in V \backslash\{z\}$. The subgroup $K(G, z)$ of the permutation group $\operatorname{Sym}(\operatorname{Rec}(G, z))$ generated by $\left\{a_{x}\right\}_{x \neq z}$ is called the sandpile group of $G$. Although the set $\operatorname{Rec}(G, z)$ depends on the choice of sink vertex, the sandpile groups for different choices of sink are isomorphic (see, e.g., 25, 27]).

The sandpile group $K(G, z)$ has a free transitive action on $\operatorname{Rec}(G, z)$, so $\# K(G, z)=\# \operatorname{Rec}(G, z)$. One can use rotor-routing to define a free transitive
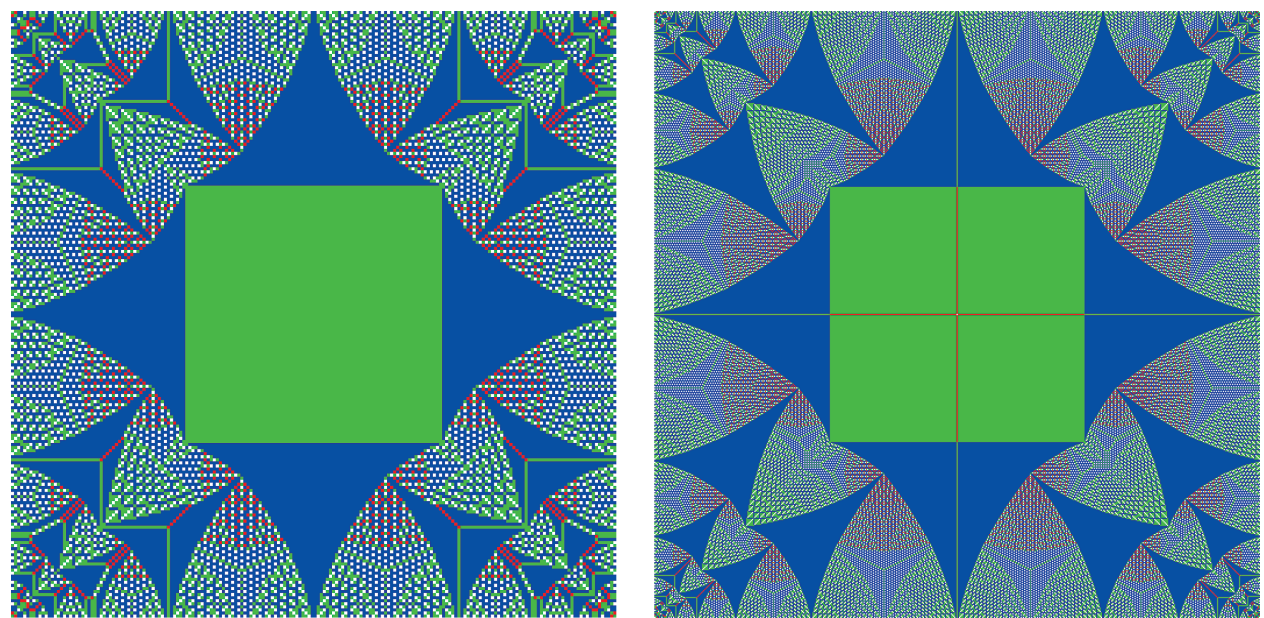

FigURE 8. Identity elements of the sandpile group $\operatorname{Rec}\left([0, n]^{2}, z\right)$ of the $n \times n$ grid graph with sink at the wired boundary (i.e., all boundary vertices are identified to a single vertex $z$ ), for $n=198$ (left) and $n=521$. 
action of $K(G, z)$ on the set of spanning trees of $G$ [25]. In particular, the number of spanning trees also equals \#K(G,z). The most important bijection between recurrent sandpiles and spanning trees uses Dhar's burning algorithm [16, 48].

A group operation $\oplus$ can also be defined directly on $\operatorname{Rec}(G, z)$, namely $s \oplus s^{\prime}$ is the stabilization of $s+s^{\prime}$. Then $s \mapsto \prod_{x} a_{x}^{s(x)}$ defines an isomorphism from $(\operatorname{Rec}(G, z), \oplus)$ to the sandpile group.

\section{LoOp erasures, Tutte POlynomial, UniCyCles}

Fix an integer $d \geq 2$. The looping constant $\xi=\xi\left(\mathbb{Z}^{d}\right)$ is defined as the expected number of neighbors of the origin on the infinite loop-erased random walk in $\mathbb{Z}^{d}$. In dimensions $d \geq 3$, this walk can be defined by erasing cycles from the simple random walk in chronological order. In dimension 2 , one first defines the loop erasure of the simple random walk stopped on exiting the box $[-n, n]^{2}$ and shows that the resulting measures converge weakly [37,39].

A unicycle is a connected graph with the same number of edges as vertices. Such a graph has exactly one cycle (Figure 9). If $G$ is a finite (multi)graph, a spanning subgraph of $G$ is a graph containing all of the vertices of $G$ and a subset of the edges. A uniform spanning unicycle (USU) of $G$ is a spanning subgraph of $G$ which is a unicycle, selected uniformly at random.

An exhaustion of $\mathbb{Z}^{d}$ is a sequence $V_{1} \subset V_{2} \subset \cdots$ of finite subsets such that $\bigcup_{n \geq 1} V_{n}=\mathbb{Z}^{d}$. Let $G_{n}$ be the multigraph obtained from $\mathbb{Z}^{d}$ by collapsing $V_{n}^{c}$ to a single vertex $z_{n}$ and removing self-loops at $z_{n}$. We do not collapse edges, so $G_{n}$ may have edges of multiplicity greater than one incident to $z_{n}$. Theorem 8.1 , below, gives a numerical relationship between the looping constant $\xi$ and the mean unicycle length

$$
\lambda_{n}=\mathbb{E}\left[\text { length of the unique cycle in a USU of } G_{n}\right]
$$

as well as the mean sandpile height

$\zeta_{n}=\mathbb{E}$ [number of particles at $\mathbf{0}$ in a uniformly random recurrent sandpile on $\left.V_{n}\right]$.

To define the last quantity of interest, recall that the Tutte polynomial of a finite (multi)graph $G=(V, E)$ is the two-variable polynomial

$$
T(x, y)=\sum_{A \subset E}(x-1)^{c(A)-1}(y-1)^{c(A)+\# A-n},
$$

where $c(A)$ is the number of connected components of the spanning subgraph $(V, A)$. Let $T_{n}(x, y)$ be the Tutte polynomial of $G_{n}$. The Tutte slope is the ratio

$$
\tau_{n}=\frac{\frac{\partial T_{n}}{\partial y}(1,1)}{\left(\# V_{n}\right) T_{n}(1,1)} .
$$

A combinatorial interpretation of $\tau_{n}$ is the number of spanning unicycles of $G_{n}$ divided by the number of rooted spanning trees of $G_{n}$.

For a finite set $V \subset \mathbb{Z}^{d}$, write $\partial V$ for the set of sites in $V^{c}$ adjacent to $V$.

Theorem 8.1 ([4]). Let $\left\{V_{n}\right\}_{n \geq 1}$ be an exhaustion of $\mathbb{Z}^{d}$ such that $V_{1}=\{\mathbf{0}\}$, $\# V_{n}=n$, and \# $\left(\partial V_{n}\right) / n \rightarrow 0$. Let $\tau_{n}, \zeta_{n}, \lambda_{n}$ be the Tutte slope, sandpile mean height, and mean unicycle length in $V_{n}$. Then the following limits exist:

$$
\tau=\lim _{n \rightarrow \infty} \tau_{n}, \quad \zeta=\lim _{n \rightarrow \infty} \zeta_{n}, \quad \lambda=\lim _{n \rightarrow \infty} \lambda_{n} .
$$




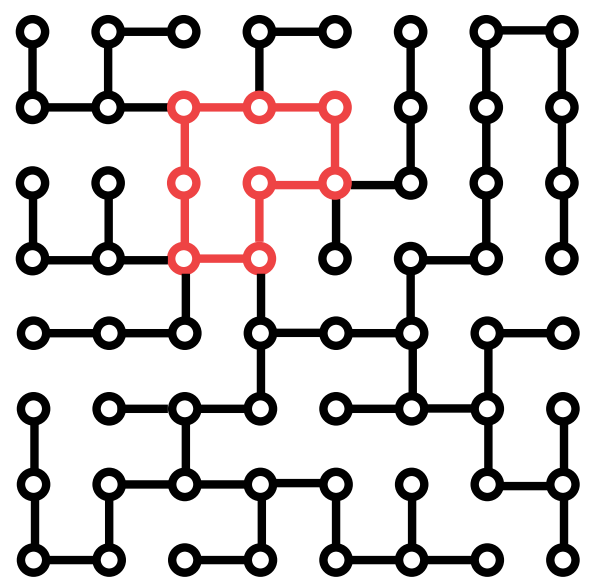

Figure 9. A spanning unicycle of the $8 \times 8$ square grid. The unique cycle is shown in red.

Their values are given in terms of the looping constant $\xi=\xi\left(\mathbb{Z}^{d}\right)$ by

$$
\tau=\frac{\xi-1}{2}, \quad \zeta=d+\frac{\xi-1}{2}, \quad \lambda=\frac{2 d-2}{\xi-1}
$$

Dimension 2 is especially intriguing, because the quantities $\xi, \tau, \zeta, \lambda$ turn out to be rational numbers.

Corollary 8.2. In the case $d=2$, we have [14,35, 62 .

$$
\xi=\frac{5}{4} \quad \text { and } \quad \zeta=\frac{17}{8}
$$

Hence by Theorem 8.1,

$$
\tau=\frac{1}{8} \quad \text { and } \quad \lambda=8
$$

The value $\zeta\left(\mathbb{Z}^{2}\right)=\frac{17}{8}$ was conjectured by Grassberger (see [17]). Poghosyan and Priezzhev [58] observed the equivalence of this conjecture with $\xi\left(\mathbb{Z}^{2}\right)=\frac{5}{4}$, and shortly thereafter three proofs [14,35, 62 appeared.

The proof that $\zeta\left(\mathbb{Z}^{2}\right)=\frac{17}{8}$ by Kenyon and Wilson [35] uses the theory of vector bundle Laplacians [34, while the proof by Poghosyan, Priezzhev, and Ruelle 62. uses monomer-dimer calculations. Earlier, Jeng, Piroux, and Ruelle [28] had reduced the computation of $\zeta\left(\mathbb{Z}^{2}\right)$ to evaluation of a certain multiple integral which they evaluated numerically as $0.5 \pm 10^{-12}$. This integral was proved to equal $\frac{1}{2}$ by Caracciolo and Sportiello [14, thus providing another proof. 
All three proofs involve powers of $1 / \pi$ which ultimately cancel out. For $i=$ $0,1,2,3$, let $p_{i}$ be the probability that a uniform recurrent sandpile in $\mathbb{Z}^{2}$ has exactly $i$ grains of sand at the origin. The proof of the distribution

$$
\begin{aligned}
& p_{0}=\frac{2}{\pi^{2}}-\frac{4}{\pi^{3}}, \\
& p_{1}=\frac{1}{4}-\frac{1}{2 \pi}-\frac{3}{\pi^{2}}+\frac{12}{\pi^{3}}, \\
& p_{2}=\frac{3}{8}+\frac{1}{\pi}-\frac{12}{\pi^{3}}, \\
& p_{3}=\frac{3}{8}-\frac{1}{2 \pi}+\frac{1}{\pi^{2}}+\frac{4}{\pi^{3}}
\end{aligned}
$$

is completed in $14,35,62$ following the work of [28, 48, 60]. In particular, $\zeta\left(\mathbb{Z}^{2}\right)=$ $p_{1}+2 p_{2}+3 p_{3}=\frac{17}{8}$.

Kassel and Wilson 33 give a new and simpler method for computing $\zeta\left(\mathbb{Z}^{2}\right)$ relying on planar duality, which also extends to other lattices. For a survey of their approach, see 32 .

These objects on finite subgraphs of $\mathbb{Z}^{d}$ also have infinite-volume limits defined on $\mathbb{Z}^{d}$ itself: Lawler [37] defined the infinite loop-erased random walk, Pemantle [53] defined the uniform spanning tree in $\mathbb{Z}^{d}$, and Athreya and Járai [5] defined the infinite-volume stationary measure for sandpiles in $\mathbb{Z}^{d}$. The latter limit uses the burning bijection of Majumdar and Dhar [48 and the one-ended property of the trees in the uniform spanning forest $\left[9,53\right.$. As for the Tutte polynomials $T_{n}$ of finite subgraphs of $\mathbb{Z}^{d}$, the limit

$$
t(x, y):=\lim _{n \rightarrow \infty} \frac{1}{n} \log T_{n}(x, y)
$$

can be expressed in terms of the pressure of the Fortuin-Kasteleyn random cluster model. By a theorem of Grimmett (see [23, Theorem 4.58]) this limit exists for all real $x, y>1$. Theorem 8.1 concerns the behavior of this limit as $(x, y) \rightarrow(1,1)$; indeed, another expression for the Tutte slope is

$$
\tau_{n}=\left.\frac{\partial}{\partial y}\left[\frac{1}{n} \log T_{n}(x, y)\right]\right|_{x=y=1} .
$$

\section{Open PROBLEMS}

We conclude by highlighting a few of the key open problems in this area.

(1) Suppose $s(x)_{x \in \mathbb{Z}^{2}}$ are independent and identically distributed random variables taking values in $\{0,1,2,3,4\}$. Viewing $s$ as a sandpile, the event that every site of $\mathbb{Z}^{2}$ topples infinitely often is invariant under translation, so it has probability 0 or 1 . We do not know of an algorithm to decide whether this probability is 0 or 1 ! See 20,41.

(2) Does the rotor-router walk in $\mathbb{Z}^{2}$ with random initial rotors (independently north, east, south, or west, each with probability $\frac{1}{4}$ ) return to the origin with probability 1? The number of sites visited by such a walk in $n$ steps is predicted to be of order $n^{2 / 3}$ [59. For a lower bound of that order, see 21. As noted there, even an upper bound of $o(n)$ would imply recurrence, which is not known! 
(3) Recall that the weak-* convergence in Theorem 6.1, proved by Pegden and Smart [54, means that the average height of the sandpile $s_{n}$ in any ball converges as the lattice spacing shrinks to zero. A natural refinement would be to show that for any ball $B$ and any integer $j$, the fraction of sites in $B$ with $j$ particles converges. Understanding the scaling limit of the sandpile identity elements (Figure 8) is another appealing problem, solved in a special case by Caracciolo, Paoletti, and Sportiello [13.

(4) As proved in [4] (and illustrated in Figure 7), the maximal elements of $\Gamma\left(\mathbb{Z}^{2}\right)$ correspond to the circles in the Apollonian band packing of $\mathbb{R}^{2}$. Because the radius and the coordinates of the center of each such circle are rational numbers, each maximal element of $\Gamma\left(\mathbb{Z}^{2}\right)$ is a matrix with rational entries. Describe the maximal elements of $\Gamma\left(\mathbb{Z}^{d}\right)$ for $d \geq 3$. Are they isolated? Do they have rational entries?

\section{About THE AUTHORS}

Lionel Levine is an assistant professor at Cornell University. He obtained his $\mathrm{PhD}$ in 2008 from the University of California, Berkeley, and was a postdoctoral scholar at MIT before moving to Cornell. He studies the dynamics of sandpile models, their generalizations (abelian networks), and scaling limits. His work uses ideas from PDE (free boundary problems), probabilistic potential theory, and combinatorics.

Yuval Peres is a principal researcher at Microsoft Research in Redmond, Washington. He obtained his $\mathrm{PhD}$ at the Hebrew University, Jersualem, in 1990 and later served on their faculty as well as the faculty at the University of California, Berkeley. He has written more than 250 research papers in probability, ergodic theory, analysis, and theoretical computer science. Peres was awarded the Rollo Davidson Prize in 1995, the Loève Prize in 2001, the David P. Robbins Prize in 2011, and was an Invited Speaker at the 2002 ICM. He is a fellow of the American Mathematical Society and a foreign associate member of the U. S. National Academy of Sciences.

\section{REFERENCES}

[1] Dov Aharonov and Harold S. Shapiro, Domains on which analytic functions satisfy quadrature identities, J. Analyse Math. 30 (1976), 39-73, DOI 10.1007/BF02786704. MR0447589

[2] Amine Asselah and Alexandre Gaudillière, From logarithmic to subdiffusive polynomial fluctuations for internal DLA and related growth models, Ann. Probab. 41 (2013), no. 3A, 11151159, DOI 10.1214/12-AOP762. MR3098673

[3] Amine Asselah and Alexandre Gaudillière, Sublogarithmic fluctuations for internal DLA, Ann. Probab. 41 (2013), no. 3A, 1160-1179, DOI 10.1214/11-AOP735. MR3098674

[4] Amine Asselah and Alexandre Gaudillière, Lower bounds on fluctuations for internal DLA, Probab. Theory Related Fields 158 (2014), no. 1-2, 39-53, DOI 10.1007/s00440-012-0476-6. MR 3152779

[5] Siva R. Athreya and Antal A. Járai, Infinite volume limit for the stationary distribution of abelian sandpile models, Comm. Math. Phys. 249 (2004), no. 1, 197-213, DOI 10.1007/s00220-004-1080-0. MR2077255

[6] László Babai, Lecture notes on the sandpile model. http://people.cs.uchicago.edu/ laci/ REU05/\#problem

[7] Per Bak, Chao Tang, and Kurt Wiesenfeld, Self-organized criticality, Phys. Rev. A (3) 38 (1988), no. 1, 364-374, DOI 10.1103/PhysRevA.38.364. MR.949160

[8] Matthew Baker and Serguei Norine, Riemann-Roch and Abel-Jacobi theory on a finite graph, Adv. Math. 215 (2007), no. 2, 766-788, DOI 10.1016/j.aim.2007.04.012. MR2355607 
[9] Itai Benjamini, Russell Lyons, Yuval Peres, and Oded Schramm, Uniform spanning forests, Ann. Probab. 29 (2001), no. 1, 1-65, DOI 10.1214/aop/1008956321. MR.1825141

[10] Anders Björner, László Lovász, and Peter W. Shor, Chip-firing games on graphs, European J. Combin. 12 (1991), no. 4, 283-291, DOI 10.1016/S0195-6698(13)80111-4. MR1120415

[11] L. A. Caffarelli, The obstacle problem revisited, J. Fourier Anal. Appl. 4 (1998), no. 4-5, 383-402, DOI 10.1007/BF02498216. MR.1658612

[12] Hannah Cairns, Some halting problems for abelian sandpiles are undecidable in dimension three. arXiv: 1508.00161

[13] Sergio Caracciolo, Guglielmo Paoletti, and Andrea Sportiello, Explicit characterization of the identity configuration in an abelian sandpile model, J. Phys. A 41 (2008), no. 49, 495003, 17, DOI 10.1088/1751-8113/41/49/495003. MR2515893

[14] Sergio Caracciolo and Andrea Sportiello, Exact integration of height probabilities in the abelian sandpile model, J. Stat. Mech. Theory Exp. 9 (2012), P09013, 14. MR2994907

[15] Joshua N. Cooper and Joel Spencer, Simulating a random walk with constant error, Combin. Probab. Comput. 15 (2006), no. 6, 815-822, DOI 10.1017/S0963548306007565. MR2271828

[16] Deepak Dhar, Self-organized critical state of sandpile automaton models, Phys. Rev. Lett. 64 (1990), no. 14, 1613-1616, DOI 10.1103/PhysRevLett.64.1613. MR.1044086

[17] Deepak Dhar, Theoretical studies of self-organized criticality, Phys. A 369 (2006), no. 1, 29-70, DOI 10.1016/j.physa.2006.04.004. MR2246566

[18] Deepak Dhar, Tridib Sadhu and Samarth Chandra, Pattern formation in growing sandpiles, Europhys. Lett. 85 (2009), 48002. arXiv:0808.1732

[19] Persi Diaconis and William Fulton, A growth model, a game, an algebra, Lagrange inversion, and characteristic classes, Rend. Sem. Mat. Univ. Pol. Torino 49 (1991), no. 1, 95-119.

[20] Anne Fey, Ronald Meester, and Frank Redig, Stabilizability and percolation in the infinite volume sandpile model, Ann. Probab. 37 (2009), no. 2, 654-675, DOI 10.1214/08-AOP415. MR2510019

[21] Laura Florescu, Lionel Levine and Yuval Peres, The range of a rotor walk, 2014. arXiv: 1408.5533

[22] Yasunari Fukai and Kôhei Uchiyama, Potential kernel for two-dimensional random walk, Ann. Probab. 24 (1996), no. 4, 1979-1992, DOI 10.1214/aop/1041903213. MR1415236

[23] Geoffrey Grimmett, The random-cluster model, Grundlehren der Mathematischen Wissenschaften [Fundamental Principles of Mathematical Sciences], vol. 333, Springer-Verlag, Berlin, 2006. MR2243761

[24] Björn Gustafsson, Quadrature identities and the Schottky double, Acta Appl. Math. 1 (1983), no. 3, 209-240, DOI 10.1007/BF00046600. MR726725

[25] Alexander E. Holroyd, Lionel Levine, Karola Mészáros, Yuval Peres, James Propp and David B. Wilson, Chip-firing and rotor-routing on directed graphs, In and out of equilibrium 2, 331-364, Progr. Probab. 60, Birkhäuser, 2008. arXiv:0801.3306

[26] Alexander E. Holroyd and James Propp, Rotor walks and Markov chains, Algorithmic probability and combinatorics, Contemp. Math., vol. 520, Amer. Math. Soc., Providence, RI, 2010, pp. 105-126, DOI 10.1090/conm/520/10256. MR2681857

[27] Antal A. Járai, Sandpile models, Cornell probability summer school lecture notes, 2014. arXiv: 1401.0354

[28] Monwhea Jeng, Geoqffroy Piroux and Philippe Ruelle, Height variables in the Abelian sandpile model: scaling fields and correlations, J. Stat. Mech. 61 (2006), 15.

[29] David Jerison, Lionel Levine, and Scott Sheffield, Logarithmic fluctuations for internal DLA, J. Amer. Math. Soc. 25 (2012), no. 1, 271-301, DOI 10.1090/S0894-0347-2011-00716-9. MR2833484

[30] David Jerison, Lionel Levine, and Scott Sheffield, Internal DLA in higher dimensions, Electron. J. Probab. 18 (2013), No. 98, 14, DOI 10.1214/EJP.v18-3137. MR.3141799

[31] David Jerison, Lionel Levine, and Scott Sheffield, Internal DLA and the Gaussian free field, Duke Math. J. 163 (2014), no. 2, 267-308, DOI 10.1215/00127094-2430259. MR3161315

[32] Adrien Kassel, Learning about critical phenomena from scribbles and sandpiles (English, with English and French summaries), Modélisation Aléatoire et Statistique-Journées MAS 2014, ESAIM Proc. Surveys, vol. 51, EDP Sci., Les Ulis, 2015, pp. 60-73, DOI 10.1051/proc/201551004. MR3440791

[33] Adrien Kassel and David B. Wilson, Looping rate and sandpile density of planar graphs, 2014. arXiv:1402.4169 
[34] Richard Kenyon, Spanning forests and the vector bundle Laplacian, Ann. Probab. 39 (2011), no. 5, 1983-2017, DOI 10.1214/10-AOP596. MR2884879

[35] Richard W. Kenyon and David B. Wilson, Spanning trees of graphs on surfaces and the intensity of loop-erased random walk on planar graphs, J. Amer. Math. Soc. 28 (2015), no. 4, 985-1030, DOI 10.1090/S0894-0347-2014-00819-5. MR3369907

[36] Gady Kozma and Ehud Schreiber, An asymptotic expansion for the discrete harmonic potential, Electron. J. Probab. 9 (2004), no. 1, 1-17, DOI 10.1214/EJP.v9-170. MR2041826

[37] Gregory F. Lawler, A self-avoiding random walk, Duke Math. J. 47 (1980), no. 3, 655-693. MR.587173

[38] Gregory F. Lawler, Subdiffusive fluctuations for internal diffusion limited aggregation, Ann. Probab. 23 (1995), no. 1, 71-86. MR 1330761

[39] Gregory F. Lawler, Intersections of random walks, Modern Birkhäuser Classics, Birkhäuser/Springer, New York, 2013. Reprint of the 1996 edition. MR2985195

[40] Gregory F. Lawler, Maury Bramson, and David Griffeath, Internal diffusion limited aggregation, Ann. Probab. 20 (1992), no. 4, 2117-2140. MR.1188055

[41] Lionel Levine, Mathav Murugan, Yuval Peres, and Baris Evren Ugurcan, The divisible sandpile at critical density, Ann. Henri Poincaré 17 (2016), no. 7, 1677-1711, DOI 10.1007/s00023015-0433-x. MR3510467

[42] Lionel Levine, Wesley Pegden, and Charles K. Smart, Apollonian structure in the Abelian sandpile, Geom. Funct. Anal. 26 (2016), no. 1, 306-336, DOI 10.1007/s00039-016-0358-7. MR3494492

[43] Lionel Levine, Wesley Pegden and Charles K. Smart, The Apollonian structure of integer superharmonic matrices, 2013. arXiv:1309.3267

[44] Lionel Levine and Yuval Peres, Strong spherical asymptotics for rotor-router aggregation and the divisible sandpile, Potential Anal. 30 (2009), no. 1, 1-27, DOI 10.1007/s11118-008-9104-6. MR2465710

[45] Lionel Levine and Yuval Peres, Scaling limits for internal aggregation models with multiple sources, J. Anal. Math. 111 (2010), 151-219, DOI 10.1007/s11854-010-0015-2. MR2747064

[46] Lionel Levine and Yuval Peres, The looping constant of $\mathbb{Z}^{d}$, Random Structures Algorithms 45 (2014), no. 1, 1-13, DOI 10.1002/rsa.20478. MR.3231081

[47] Dino J. Lorenzini, Arithmetical graphs, Math. Ann. 285 (1989), no. 3, 481-501, DOI 10.1007/BF01455069. MR1019714

[48] S. N. Majumdar and Deepak Dhar, Height correlations in the Abelian sandpile model, J. Phys. A: Math. Gen. 24 (1991), no. 7, L357.

[49] Madhusudan Manjunath and Bernd Sturmfels, Monomials, binomials and Riemann-Roch, J. Algebraic Combin. 37 (2013), no. 4, 737-756, DOI 10.1007/s10801-012-0386-9. MR3047017

[50] Fatemeh Mohammadi and Farbod Shokrieh, Divisors on graphs, connected flags, and syzygies, Int. Math. Res. Not. IMRN 24 (2014), 6839-6905. MR3291642

[51] Paul Meakin and J. M. Deutch, The formation of surfaces by diffusion-limited annihilation, J. Chem. Phys. 85 (1986), 2320.

[52] Cristopher Moore and Martin Nilsson, The computational complexity of sandpiles, J. Statist. Phys. 96 (1999), no. 1-2, 205-224, DOI 10.1023/A:1004524500416. MR.1706761

[53] Robin Pemantle, Choosing a spanning tree for the integer lattice uniformly, Ann. Probab. 19 (1991), no. 4, 1559-1574. MR 1127715

[54] Wesley Pegden and Charles K. Smart, Convergence of the Abelian sandpile, Duke Math. J. 162 (2013), no. 4, 627-642, DOI 10.1215/00127094-2079677. MR3039676

[55] Wesley Pegden, Sandpile galleries. http://www.math.cmu.edu/ wes/sandgallery.html

[56] David Perkinson, Jacob Perlman, and John Wilmes, Primer for the algebraic geometry of sandpiles, Tropical and non-Archimedean geometry, Contemp. Math., vol. 605, Amer. Math. Soc., Providence, RI, 2013, pp. 211-256, DOI 10.1090/conm/605/12117. MR3204273

[57] David Perkinson and Bryan Head, SandpilesApp. http://people.reed.edu/ davidp/sand/ program/program.html

[58] V. S. Poghosyan and V. B. Priezzhev, The problem of predecessors on spanning trees, Acta Polytechnica, 51 (2011), 1, arXiv: 1010.5415

[59] A. M. Povolotsky, V. B. Priezzhev, and R. R. Shcherbakov, Dynamics of Eulerian walkers, Physical Review E 58 (1998), no. 5, 5449.

[60] V. B. Priezzhev, Structure of two dimensional sandpile. I. Height probabilities, J. Stat. Phys., 74 (1994), 955-979. 
[61] V. B. Priezzhev, Deepak Dhar, Abhishek Dhar and Supriya Krishnamurthy, Eulerian walkers as a model of self-organised criticality, Phys. Rev. Lett. 77 (1996), 5079-5082. arXiv: cond-mat/9611019

[62] V. S. Poghosyan, V. B. Priezzhev and P. Ruelle, Return probability for the loop-erased random walk and mean height in the Abelian sandpile model: a proof. Journal of Statistical Mechanics: Theory and Experiment 10 (2011), P10004.

[63] James Propp, Random walk and random aggregation, derandomized, 2003. http://research. microsoft.com/apps/video/default . aspx?id=104906.

[64] Makoto Sakai, Quadrature domains, Lecture Notes in Mathematics, vol. 934, Springer-Verlag, Berlin-New York, 1982. MR663007

[65] Makoto Sakai, Solutions to the obstacle problem as Green potentials, J. Analyse Math. 44 (1984/85), 97-116, DOI 10.1007/BF02790192. MR801289

[66] Frank Spitzer, Principles of random walk, 2nd ed., Graduate Texts in Mathematics, Vol. 34, Springer-Verlag, New York-Heidelberg, 1976. MR0388547

[67] Kôhei Uchiyama, Green's functions for random walks on $\mathbf{Z}^{N}$, Proc. London Math. Soc. (3) 77 (1998), no. 1, 215-240, DOI 10.1112/S0024611598000458. MR.1625467

[68] Israel A. Wagner, Michael Lindenbaum, and Alfred M. Bruckstein, Smell as a computational resource-a lesson we can learn from the ant, Israel Symposium on Theory of Computing and Systems (Jerusalem, 1996), IEEE Comput. Soc. Press, Los Alamitos, CA, 1996, pp. 219-230. MR.1436463

Department of Mathematics, Cornell University, Ithaca, New York 14853

$U R L:$ http://www . math. cornell.edu/ levine

Microsoft Research, Redmond, Washington 98052

$U R L:$ http://research.microsoft.com/en-us/um/people/peres/ 